\title{
Real-time simulation of interferometric gravitational wave detectors involving moving mirrors
}

\author{
Biplab Bhawal \\ Groupe VIRGO, Laboratoire de l'Accélérateur Linéaire, Centre National de la Recherche Scientifique IN2P3, \\ Bâtiment 208, Orsay Cedex, 91405, France
}

Received January 31, 1997; accepted June 20, 1997; revised manuscript received July 3, 1997

\begin{abstract}
A method of real-time dynamical simulation for laser interferometric gravitational wave detectors is presented. The method is based on a digital filtering approach and a number of important physical points understood by a step-by-step investigation of two-mirror cavities, a three-mirror coupled cavity, and a full-length power-recycled interferometer with mirrors having longitudinal motion. The final analytical representation used for the fast simulation of a full-length power-recycled interferometer is analogous to a two-mirror dynamical cavity with time-dependent reflectivities, when intracavity fields of the interferometer are expressed together in a state-vector representation. A detailed discussion establishes the relationships among physical effects pertaining to field evolution in two-mirror cavities and coupled cavities or to the full interferometer. (C) 1998 Optical Society of America [S0740-3232(97)01312-4]
\end{abstract}

OCIS codes: $120.3180,120.2230$.

\section{INTRODUCTION}

Gravitational wave detectors based on laser interferometry are currently being developed by various collaborating groups. ${ }^{1}$ Some features common to the long-baseline facilities (as shown in Fig. 1) are these: (a) to increase the storage time of light, Fabry-Perot (FP) cavities are used in the arms; (b) to maximize the signal-to-noise ratio, the arm length difference is adjusted so that the output port remains on the dark fringe; (c) to reduce the shot noise, a high-power laser is used, in conjunction with the power-recycling technique, ${ }^{2}$ in which, with a dark-fringe operation, the outgoing light is recycled back into the interferometer by putting a mirror in front of the source, thus enhancing the laser power; (d) to isolate mirrors from the seismic noise, all six mirrors are suspended as pendulums.

The full interferometer is thus a system of coupled cavities. In addition to maintaining the dark-fringe condition, all of these cavities are to be kept on resonance with the laser source. This is a difficult task, since the suspended mirrors, by becoming excited by the residual seismic noise, may oscillate around their equilibrium points at very low frequency with an amplitude of some tens of wavelengths.

All these features provide newer dimensions to the interferometry of the gravitational wave detectors. It should be noted that the physical effects related to dynamical single or coupled cavities and interferometers have hardly been discussed in the literature because of the simple reason that before gravitational wave detectors were conceived, people could not even imagine designing such a system for some useful purpose.

A detailed investigation of these effects is necessary for a complete understanding of the operation of interfero- metric detectors. At this time, it is therefore extremely important to develop numerical simulation programs that can predict the behavior of the detector with sufficient accuracy and aid in evaluating and optimizing parameters of different components of the interferometer. We need to know how fields at various locations of the interferometer change as the mirrors move so that we can use this information to detect any variation in the required operating condition. An automatic length-control system based on these dynamical parameters can then be developed.

It is straightforward to write a simulation program by using exact equations of field evolution, but in comparison with real time, the computational time of such a simulation is too long. Therefore the exact simulation is not of much use for either the control system or investigation of the physical effects. Thus it is necessary to develop fast codes for all or part of the interferometer. If the behavior of various servo responses can be properly ascertained, we can also compare performances of the real interferometer with the one running in the computer (provided the latter is fast enough) and initiate proper action if anything goes wrong during the operation. Such a complete simulation that incorporates both the signal generated by moving mirrors in a high-finesse two-mirror cavity and the response of the servo control system has been reported by the LIGO team. ${ }^{3}$

With these objectives in mind, in this paper I develop a method of dynamical simulation for a power-recycled interferometer that is able to perform real-time calculations at various levels of accuracy. The method is based on a digital filtering approach and a number of important physical points understood by use of a step-by-step investigation process. This investigation finally establishes a 


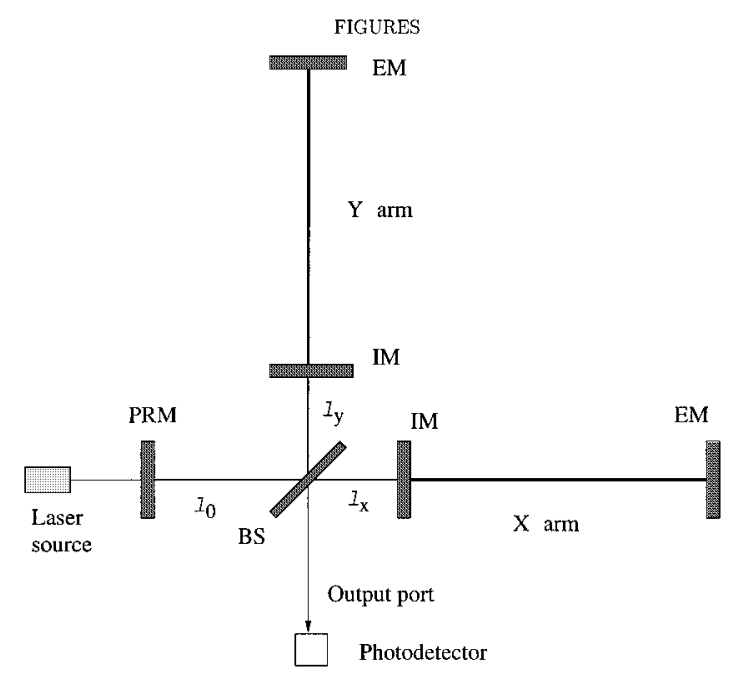

Fig. 1. Configuration of a power-recycled interferometer. BS, beam splitter; EM, end mirror; IM, input mirror; PRM, powerrecycling mirror.

relationship among characteristics of dynamical field evolution in a long-baseline interferometer and those in twomirror and three-mirror coupled cavities. Therefore in Sections 2, 3, and 4, I introduce various aspects of this method and discuss the characteristics of the dynamical response in a low-finesse two-mirror cavity, a high-finesse two-mirror cavity, and a three-mirror coupled cavity, respectively. The special features of coupling between recycling cavity and arm cavity are discussed in Section 5 with the help of results obtained by performing computer experiments. Finally, in Section 6 I use all these understandings along with the techniques developed therefrom to write the fast numerical code for a power-recycled interferometer. The physical effects related to the coupling of fields in between two arm cavities of an interferometer are also discussed in Section 6. Section 7 summarizes important conclusions about the physical effects in a dynamical power-recycled interferometer as well as considerations regarding the computational speed of the codes developed.

\section{FAST SIMULATION OF LOW-FINESSE TWO-MIRROR CAVITIES: DIGITAL FILTERING APPROACH BASED ON THE PERTURBATION METHOD}

In this section I study the simplest case of simulation, i.e., that of a two-mirror cavity of low finesse. Such a cavity of finesse $\sim 50$ will be used in the arms of the VIRGO interferometer. ${ }^{4}$ We may note in advance that a direct analogy can be established between the response of a power-recycled interferometer and that of a two-mirror cavity under general dynamical conditions, as will be shown in Section 6. The dynamical response may show low- or high-finesse characteristics depending on the operating condition of the light beam. Results of the investigation in this section are therefore very important and will be applied in writing the simulation program of the whole interferometer in Section 6.

An analogy is established here between a digital filter and the linear response of a cavity to small motion of mir- rors. Then a fast simulation procedure based on this analogy is developed. ${ }^{5}$ The analytical calculation presented here for the response of a low-finesse cavity is based on the perturbative technique. I investigate how well such a perturbative calculation can describe the evolution of fields when used in the fast dynamical simulation procedure based on the digital filtering approach (DFA). The numerical results and a comparison with other methods are also presented.

\section{A. Cavity Linearized Equation}

Let us first write the exact equation for the intracavity field $F$ based on the notation of Fig. 2 by taking the laser source to be the reference point for the motion of the mirrors:

$$
\begin{aligned}
F(t)= & t_{c} A \exp j\left[\frac{2 \pi}{\lambda} x_{c}(t)\right]+r_{c} r_{e} \\
& \times \exp j[\theta(t-\tau / 2)] F(t-\tau),
\end{aligned}
$$

where $\tau=2 L / c=2 \times 10^{-5} \mathrm{~s}$ is the round-trip time for VIRGO $3-\mathrm{Km}$ cavities and $\lambda$ is the wavelength of the laser light, $1.064 \mu \mathrm{m} ; t_{c}$ is the amplitude transmittivity of the input mirror; and $r_{c}$ and $r_{e}$ are the amplitude reflectivities of the input mirror and the end mirror, respectively. The quantity $\theta(t)$ represents the round-trip phase offset in the cavity at any time $t$ :

$$
\theta(t-\tau / 2)=\phi+\frac{2 \pi}{\lambda} 2 x(t-\tau / 2)
$$

where $\phi$ is the initial (constant) round-trip phase offset and $x(t-\tau / 2)$ is the variation in the length of the cavity as experienced by the light at time $t$ owing to movement of the mirrors:

$$
2 x(t-\tau / 2)=-x_{c}(t-\tau)+2 x_{e}(t-\tau / 2)-x_{c}(t),
$$

where $x_{c}(t)$ and $x_{e}(t)$ are displacements of the input and the end mirror, respectively.

If we assume that both the variation $x(t)$ of the length $L$ of the cavity and the frequency of such variation are small enough, we can reasonably predict that the field amplitudes will also vary slowly around some stationary point (an elaborate and nicer treatment can be found in Ref. 6). Equation (1) can therefore be written in the following form for small $x$ :

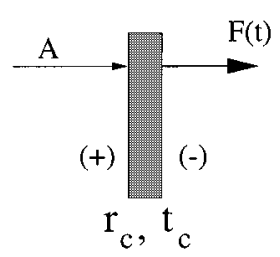

Input Mirror

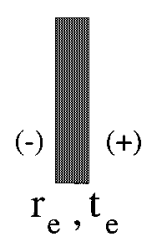

End Mirror
Fig. 2. Notation for a two-mirror cavity; $r_{c}\left(r_{e}\right)$ and $t_{c}\left(t_{e}\right)$ are amplitude reflectivity and transmittivity, respectively, for the input (end) mirror. The sign (+) or ( - ) indicates the phase factor acquired on reflection from that side of the mirror. 


$$
\begin{aligned}
F(t)=t_{c} A & \exp j\left[\frac{2 \pi}{\lambda} x_{c}(t)\right] \\
+ & R\left[1+j \frac{2 \pi}{\lambda} 2 x\left(t-\frac{\tau}{2}\right)\right] F(t-\tau),
\end{aligned}
$$

where

$$
R=r_{c} r_{e} \exp j(\phi) .
$$

Note that we make a simplifying assumption that the input light $A$ is constant. The stationary point of the intracavity field can thus be written from the zeroth-order equation:

$$
F_{0}=\frac{t_{c} A}{1-R}
$$

which simply represents the quasi-static field, i.e., when mirrors do not move at all or move so slowly that the fields do not become affected for a long time. The variation of the intracavity light from this can be obtained from the first-order equation:

$$
\delta F(t)=R\left[\delta F(t-\tau)+j \frac{4 \pi}{\lambda} F_{0} x\left(t-\frac{\tau}{2}\right)\right] .
$$

This leads to the following transfer function for the cavity in the $s$ domain of the Laplace transformation:

$$
H_{c}(s)=\frac{\delta F(s)}{x(s)}=j P F_{0} \frac{\exp \left(-\frac{\tau}{2} s\right)}{1-R \exp (-\tau s)} x(s),
$$

where

$$
P=(4 \pi / \lambda) R \text {. }
$$

Equation (8), in itself, is not of much use for the timedomain simulation, since we need to know $x(t)$ in the full time domain $(t \rightarrow+\infty)$ for the calculation of $\delta F(s)$. However, as is discussed in Subsection 2.B, one can appropriately apply the technique of digital filtering to take advantage of linear equation (7) in order to develop a fast computational method for the evolution of light fields.

\section{B. Cavity As a Digital Filter}

A number of standard texts ${ }^{7}$ on digital filters are available. However, it is worth introducing a few important concepts here to help us understand the domain of validity of the analogy between a cavity and a digital filter.

Let us consider a transfer function of the following form corresponding to the input $X$ and output $Y$ in the $s$ domain:

$$
H(s)=\frac{Y(s)}{X(s)}=\frac{\sum_{k=0}^{K} C_{k} \exp (-k s \Delta)}{1-\sum_{m=1}^{M} D_{m} \exp (-m s \Delta)},
$$

where $k$ and $m$ are integers and $\Delta$ is some fixed time interval. The correspondence between this transfer function and its own discrete form in the $Z$-transform domain can be established by a conformal transformation, $z$ $=\exp (s \Delta)$ :

$$
\bar{H}(n)=\frac{\bar{Y}(n)}{\bar{X}(n)}=\frac{\sum_{k=0}^{K} C_{k} z^{-k}}{1-\sum_{m=1}^{M} D_{m} z^{-m}},
$$

where $n$ represents the sampled points with a time period $\Delta$. So one can now arrive at the following equation describing the output function in its discrete form:

$$
\bar{Y}(n)=\sum_{k=0}^{K} C_{k} \bar{X}(n-k)+\sum_{m=1}^{M} D_{m} \bar{Y}(n-m) .
$$

This is known as the infinite impulse response (IIR) filter, which is actually a series of feedback loops, as is obvious from Eq. (12)

Let us now look back at Eq. (8) and compare it with Eqs. (11) and (12). We can easily see that Eq. (8) can provide only two nonzero coefficients $\left(C_{1}=P F_{0}\right.$ and $D_{2}$ $=r e^{j \phi}$ ) for the evaluation of the output, i.e., for the variation in the intracavity light, $\delta F$, in the discrete time domain with a sampling period of $\tau / 2$. However, if we wish, we can always increase the number of coefficients $C_{k}$ simply by explicitly evolving the recursive relation, Eq. (7), in $N$ steps for time $N \tau$. In optical language, this is equivalent to tracing the path of the light for $N$ number of bounces on the moving end mirror.

It should be noted that such a procedure, however, does not increase the number of nonzero $D_{m}$ coefficients and in fact reduces the numerical importance of the only available coefficient (which is $D_{m}$ for $m=2 n$ at any stage $n$ ) at every step of this evolution by a factor $R$. So if we are able to take a sufficiently large value of $N$, we can reasonably neglect the contribution of previous outputs in the present value of the output in a low-finesse cavity. Such a filter, which does not receive any input in the form of a feedback, is called a finite impulse response (FIR) filter, and for such filters the coefficients $C_{k}$ represent the unity impulse response of the filter. It should be noted that by going through this step, we converted the concept of the cavity as a filter from an IIR filter one to a FIR one.

In the context of a two-mirror cavity, we can thus write the following equation for the intracavity light directly from Eq. (7):

$$
F(t+N \tau)=\frac{t_{1} A \exp j\left[x_{c}(t)\right]}{1-R}(1+j P \text { sum }),
$$

where

$$
\operatorname{sum}=\sum_{n=1}^{N} R^{N-n} x\left[t+\left(n-\frac{1}{2}\right) \tau\right] .
$$

The structure of the correction term shows that it is merely the time convolution of the input $x(n)$ with the unity impulse response function represented by the coefficients

$$
C_{k}=j P F_{0} R^{k-1}, \quad k=1,2,3, \ldots .
$$

We should keep in mind that the usefulness of the analysis presented here depends strongly on the validity of the assumption that the mirror movement is very slow, which enables us to use the linear equation for the cavity. 
So the validity of the analysis depends on how we make a suitable choice for the number of bounces $N$. In Subsection 2.C, we shall ascertain the value of $N$ numerically by taking error considerations into account.

\section{Simulation and Results}

The numerical investigation shows that the phase factor that depends on $x_{c}(t)$ in Eq. (13) has almost no influence on the evolution of fields in a low-finesse cavity (note that this will not be so in Section 3, where we discuss highfinesse cavities). So, while developing the following simulation technique, we can safely ignore this factor. This also means that for a low-finesse cavity, it does not matter whether we take the laser source or the input mirror to be our reference point of mirror motion, provided that the speed of the input mirror is of the order of $1 \mu \mathrm{m} / \mathrm{s}$ or less. lows:

The procedure of the numerical simulation is as fol-

- Step 1: Time is sliced into equal intervals of width $\Delta=N \tau$, so that any time $t_{i}=i N \tau$, where $i$ is an integer.

- Step 2: The phase $\phi$ is fixed to the following value during any time interval $\left(t_{i}, t_{i+1}\right)$ :

$$
\phi_{i}=\phi_{0}+\frac{4 \pi}{\lambda} x(i \Delta),
$$

where $\phi_{0}$ is a small initial phase offset at time $t=0$.

- Step 3: During the time interval $\left(t_{i}, t_{i+1}\right)$, the rate of change of the cavity length is also assumed to be constant:

$$
w_{i}=\frac{x_{i+1}-x_{i}}{N \tau}
$$

This is a valid assumption, as long as the frequency of the mirror oscillation is small and we choose a reasonable value of $N$. For example, for a frequency of, say, 200 $\mathrm{mHz}$, we can reasonably talk about a value of $N$ up to, say, 200 without making a significant error.

- Step 4: The assumption in step 3 enables us to arrive at a very simple expression for the convolution sum in Eq. (13). This sum now turns out to be an arithmetico-geometric series, which can be easily converted into its compact form:

$\operatorname{sum}=\frac{\left(N-\frac{1}{2}\right)-\left(N+\frac{1}{2}\right) r_{e} r_{c} \exp j\left(\phi_{i}\right)}{\left[1-r_{c} r_{e} \exp j\left(\phi_{i}\right)\right]^{2}} w_{i} \tau+\mathscr{O}\left(R^{N}\right)$.

The $\mathscr{Q}\left(R^{N}\right)$ terms can be neglected for sufficiently large values of $N$.

- Step 5: Substituting $i \leftarrow i+1$, the procedure is repeated, i.e., the values of $\phi_{i}$ and $w_{i}$ are changed in the next step and $F\left(t_{i+1}\right)$ is calculated.

As one can see, this method makes a slope-by-slope approximation of the actual mirror movement, where each slope (velocity $w_{i}$ ) lasts for a short time. The span of this short time (represented by $N$ ) can be determined by numerical investigation of the error level introduced by slope-by-slope approximation.

The above method based on the DFA can now be compared with other methods ${ }^{8}$ of simulation that have been tried in order to obtain a fast simulation of a low-finesse cavity: (a) the sequential method (SM), which computes the intracavity beam at each round-trip and so is accurate but very slow, (b) the quasi-static method (QSM), which computes a stationary value from the cavity Airy function [Eq. (6)] for the intracavity light on the assumption of constancy of all quantities over a sufficiently long time and whose error is thus independent of the time step $N$ used in computation, (c) the differential equation method ${ }^{8}$ (DEM) which, under the assumption of constancy of $x$ (and thus of the phase offset) over an interval $\Delta=N \tau$, solves the cavity differential equation in that interval, sets the initial condition for the next step with that solution, changes the phase by another step, and solves the differential equation again. As a result, for smaller values of $N$ the DEM approaches the accurate SM, and for higher values of $N$ it approaches the QSM.

An important difference between the DEM method and the present method is that whereas the former makes a step-by-step approximation (i.e., $x$ is constant over the interval $\Delta$ ) for the mirror displacement $x(t)$, the present method takes a slope-by-slope approach (i.e., $x$ changes with constant velocity during $\Delta$ ). As a result, a comparison between either the DEM or the QSM and the present method can be made only for small values of $N$. For large values of $N$, such a comparison is meaningless, since in that case only in the limit $w \rightarrow 0$ can the present method be expected to be similar to either the QSM or the DEM.

The simulation program as described in the last subsection is run for two values of $w$ (which, for the sake of simplicity, has been assumed to be constant throughout the time of the simulation), $1 \mu \mathrm{m} / \mathrm{s}$ and $0.5 \mu \mathrm{m} / \mathrm{s}$. The values of $r_{c}$ and $r_{e}$ are chosen to be $94.0 \%$ and $99.99 \%$, respectively, which corresponds to a finesse of approximately 50. A power loss of 10 ppm has been considered for both mirrors.

A typical resonance curve generated by the exact SM method for such a cavity with $w=1 \mu \mathrm{m} / \mathrm{s}$ is shown in Fig. 3. The resonance curve generated by the QSM, the DEM, or the DFA under similar conditions appears to be similar to this curve, but any of these methods actually makes some error (with respect to SM), which remains invisible by the resolution of the plot. The relative error made by either the QSM or the DFA with respect to the accurate SM method in drawing the same resonance curve is plotted in Fig. 4. The relative error of some method $Y$ with respect to some other method $X$ is defined as follows:

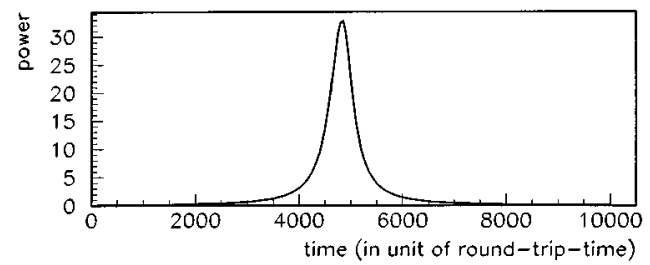

Fig. 3. Typical resonance curve for a low-finesse cavity that can be drawn by using the SM, QSM, DEM, or DFA (with reasonable values of $N$ in the last two cases), while the cavity length changes at a rate $w=1 \mu \mathrm{m} / \mathrm{s}$. Input power $|A|^{2}$ is one unit. Note that the resolution of the plot cannot make any difference among curves obtained from these methods. 
relative $\operatorname{error}(\%)$

$$
\frac{\text { value predicted by } Y-\text { value given by } X}{\text { value given by } X} \times 100 \text {. }
$$

The nature of the error curve for the DFA as shown in Fig. 4(b) is more or less similar for various values of $N$ and $w$ but is quite different from those for either the QSM [Fig. 4(a)] or the DEM. The error curves generated by the QSM and the DEM are similar looking, but the level of error is less with the DEM than with the $\mathrm{QSM}^{8}$ ); the error level of the DFA is the least among these methods.

The maximum values of the error curves of the DFA as a function of $N$ are plotted in Fig. 5. One can see that the
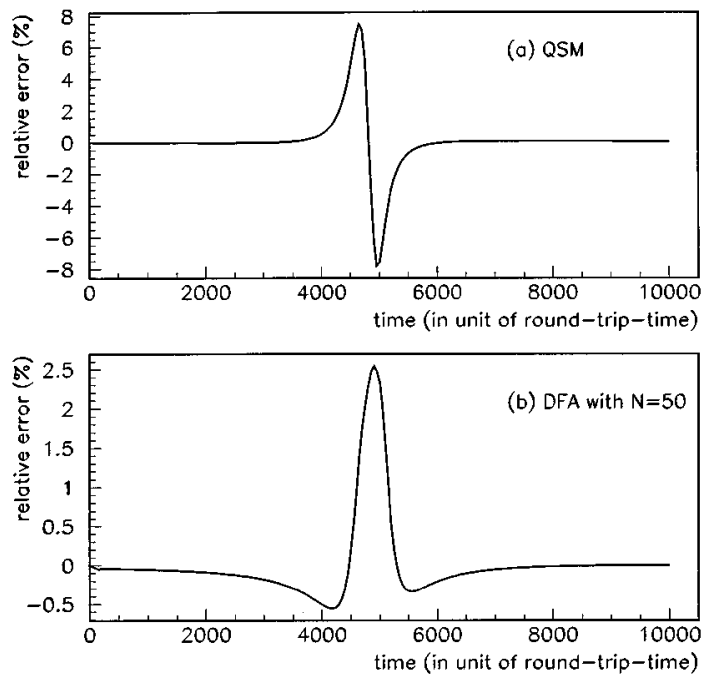

Fig. 4. Relative error (\%) of (a) QSM and (b) DFA based on perturbative calculation in comparison with the exact SM in computing the resonance curve of Fig. 3.

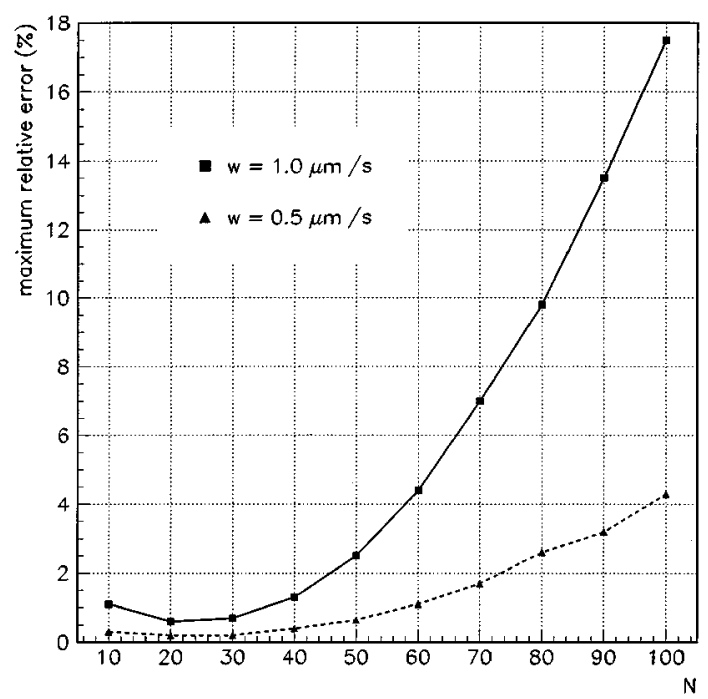

Fig. 5. Maximum values of the relative error (\%) of the DFA based on perturbative calculation in comparison with the exact SM (in computing the resonance curve of Fig. 3) plotted against $N$, the number of steps for two rates of change in the cavity length.
Table 1. Approximate Values of the Peaks of the Relative Error Curves for Cavities with Various Values of the Finesse, with $\boldsymbol{N}$ fixed to 50

\begin{tabular}{|c|c|c|}
\hline$r_{c}$ & $\begin{array}{c}\text { Maximum } \\
\text { Relative Error }(\%) \\
(w=0.5 \mu \mathrm{m} / \mathrm{s})\end{array}$ & $\begin{array}{c}\text { Maximum } \\
\text { Relative Error }(\%) \\
(w=1 \mu \mathrm{m} / \mathrm{s})\end{array}$ \\
\hline 0.94 & 0.65 & 2.5 \\
\hline 0.95 & 0.80 & 3.2 \\
\hline 0.96 & 1.0 & 4.2 \\
\hline 0.97 & 2.0 & 8.0 \\
\hline 0.975 & 4.0 & 14.5 \\
\hline 0.98 & 10.0 & 32.0 \\
\hline 0.985 & 30.0 & 90.0 \\
\hline 0.99 & 175.0 & 325.0 \\
\hline
\end{tabular}

simulation performs quite satisfactorily even for large values of $N$ (=say, 60 for $w=1 \mu \mathrm{m} / \mathrm{s}$ or, say, 100 for $w$ $=0.5 \mu \mathrm{m} / \mathrm{s}$, if we take an arbitrary value of $\sim 4 \%$ to be an upper limit). I neglected $\mathscr{O}\left(r^{N}\right)$ terms in Eq. (18) for simulation, and thus for sufficiently low value of $N$ the maximum value of error increases again.

The relative error increases not only for higher values of $N$ and mirror velocity but also for higher values of the cavity finesse. The finesse of a cavity can also be expressed by the product of the amplitude reflectivities of the two mirrors. Since we always keep the value of $r_{e}$ equal to a constant, $99.99 \%$, from now on I will always refer to the finesse of a cavity in terms of the reflectivity of the input mirror, $r_{c}$.

Now we fix $N=50$ and tabulate the maximum values of error curves generated by the DFA for increasing values of $r_{c}$ in Table 1 . As we can see, the error increases too fast just after crossing the value $r_{c}=0.975$. A validity domain for this method based on perturbative calculations may be ascertained in terms of an upper limit of $\sim 0.975$ on $r_{c}$ for a mirror velocity of $\leqslant 1 \mu \mathrm{m} / \mathrm{s}$; This limit, however, depends on what trade-off one would like to make between computational speed and error in its application and should be mentioned in that spirit. But whatever be the trade-off, the perturbative calculation of the cavity response is certainly not applicable in the DFA of fast simulation for a value of finesse corresponding to $r_{c}$ $\geqslant 0.99$

From now on, I refer to cavities with $r_{c} \leqslant 0.975$ as lowfinesse cavities and those with $r_{c} \geqslant 0.985$ as high-finesse cavities. The cavities with $0.975<r_{c}<0.985$ correspond to the intermediate range for which a perturbative approach of simulation leads to too much error for high values of mirror velocities (i.e., $\approx 1 \mu \mathrm{m} / \mathrm{s}$ ) but, on the other hand, for which the high-finesse characteristics of the cavity response (as I discuss in Section 3) are not so prominent. In any case, the fast simulation methods to be developed in Section 3 for high-finesse cavities can always be applied to lower-finesse cavities. I am making this division in the range of finesse just to make a clearcut distinction between the high-finesse and the lowfinesse characteristics, which allows us a convenient basis for our discussion. 


\section{SIMULATION OF HIGH-FINESSE TWO- MIRROR CAVITIES: DIGITAL FILTERING APPROACH BASED ON THE JUMP-AND-SUMP METHOD}

We may note again in advance that there exists a direct analogy between the dynamical response of a powerrecycled interferometer and that of a two-mirror cavity. We will also see in Section 6 that most of the time in a general dynamical situation and especially at the desired operating condition of the laser light, the response of the interferometer shows high-finesse characteristics. The results of Section 2 indicate that the perturbative approach of performing cavity calculations is not suitable for describing the enhanced sensitivity of the high-finesse dynamical cavities to small perturbations in the form of mirror movement. In this section I introduce a method ${ }^{9}$ that replaces the perturbative calculation in the framework of the DFA.

The enhanced sensitivity of the cavity in its dynamical response to mirror movement can be illustrated here by drawing a resonance curve for the intracavity field by using Eq. (1) of the SM method [Fig. 6(a)] when only the end mirror moves at a speed $1 \mu \mathrm{m} / \mathrm{s}$. The value of $r_{c}$ is chosen to be 0.998733 . (The question "why such a value?" will get answered in Section 6, where we will see that this choice reduces the number of figures in this paper).

The corresponding resonance curve as generated by the QSM method when the mirror movement is very slow or zero is shown in Fig. 6(b). A comparison of Figs. 6(a) and 6 (b) with all those generated for the low-finesse cavity in Section 2 reveals certain special characteristics of the dynamical response of high-finesse cavities:

(a) Failure to achieve maximum peak value: The peak value of the dynamical curve fails to reach the maximum value of power [the quasi-static value, Fig. 6(b)] that can be achieved on resonance from such a system. The
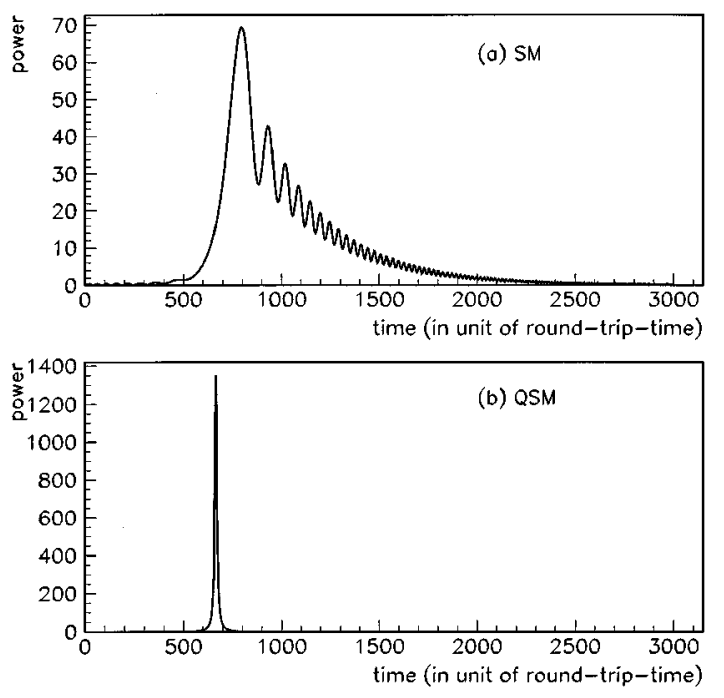

Fig. 6. (a) Dynamical resonance curve for a high-finesse $\left(r_{c}\right.$ $=0.998733$ ) two-mirror cavity while only the end mirror moves and so the cavity length changes at a rate of $w=1 \mu \mathrm{m} / \mathrm{s}$. Input power is one unit. (b) Quasi-static resonance curve for the same cavity.

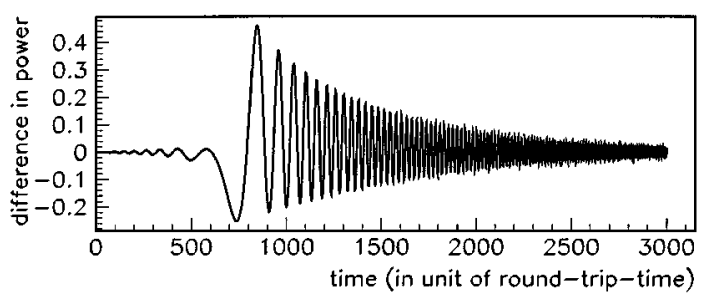

Fig. 7. Difference in power between the case when the input mirror moves (at a speed of $1 \mu \mathrm{m} / \mathrm{s}$ ) and when it does not but the rate of change in cavity length $w$ is the same in both cases $(=1 \mu \mathrm{m} / \mathrm{s})$. The latter case corresponds to Fig. 6(a).

reason is the fast motion of mirror, which does not allow partial beams to stick to the resonance or near-resonance value for a long time.

(b) Displacement of the peak from the resonance point: The peak value of the dynamical resonance curve appears a few round-trips after the actual point of resonance (i.e., $\theta=2 \pi)$. As a result of high finesse, a large number of partial beams get stored for a long time, each of them bearing different phase information about the moving cavity. A combination of all these beams finds a maximum point of their combined power at a point somewhat displaced in time from their quasi-static resonance points.

(c) Oscillations after crossing the peak: The oscillation of power just after the peak is crossed is due to the generation of beats by the interference of the Dopplershifted beams that were there for a long time (as a result of high finesse) and those beams that are relatively newcomers. The frequency of this oscillation increases for a short time and then again decreases to zero as the cavity goes farther from resonance and the contribution of longlasting beams becomes less and less. (The last point can be shown in a frequency-domain simulation. It will also be evident when we study the relative error in the resonance curves generated by some nonexact equations at the end of this section).

(d) Enhanced sensitivity toward the movement of the input mirror: Unlike in the low-finesse case, the field evolution now is affected by the Doppler shift that is experienced by the input light with respect to the source as a result of the movement of the input mirror. Figure 7 shows the difference in power of the field $F$ between a case when both mirrors move and when only the end mirror moves but the rate of change of the cavity length $w$ is kept the same $(=1 \mu \mathrm{m} / \mathrm{s})$ in both cases. The resonance curve corresponding to the latter case is already shown in Fig. 6(a). The numerical investigation and Fig. 7 show that if the mirror velocity is restricted to $\leqslant 1 \mu \mathrm{m} / \mathrm{s}$, there will be little and insignificant effect on the field evolution near resonance. We can thus neglect this phase factor in the input light again for the case of high-finesse cavities without committing significant error.

To describe this enhanced sensitivity with a faster simulation, we incorporate the following analytical calculation into the DFA fast simulation, replacing the perturbative method:

- Step 1: Let us start with the following equation of the intracavity field $F$, 


$$
F(t+\tau)=t_{c} A+R \exp j\left[\frac{4 \pi}{\lambda} x(t+\tau / 2)\right] F(t),
$$

written for a small variation $x$ in the length of the cavity. This equation is then recursively evolved in time for $N$ steps, where one step is equivalent to the round-trip time $\tau$ of the cavity, and thus we obtain

$$
\begin{aligned}
F(t+N \tau)= & t_{c} A\left(1+\sum_{n=2}^{N} R^{n-1} \prod_{k=N-n+2}^{N} \exp _{k}\right) \\
& +F(t) R^{N} \prod_{k=1}^{N} \exp _{k},
\end{aligned}
$$

where

$$
\begin{aligned}
\exp _{k} & =\exp \left(j \frac{4 \pi}{\lambda} x_{k}\right), \\
x_{k} & \equiv x(t+(k-1 / 2) \tau),
\end{aligned}
$$

which is described by Eq. (3).

- Step 2: Let us assume that for a reasonable number of steps $N$, the rate of change in cavity length can be approximated by a constant $w$, which permits Eq. (22) to be written as

$$
\exp _{k}=\exp [\xi(k-1 / 2)],
$$

where $\xi=j 4 \pi w \tau / \lambda$.

- Step 3: It is also assumed that the mirror displacement that takes place within the interval $N \tau$ is very small, so that we can linearize all the exponentials in Eq. (21), thus obtaining

$$
\begin{aligned}
F(t+N \tau)= & \frac{t_{c} A\left(1-R^{N}\right)}{1-R}+t_{c} A \xi R \sum_{i=1}^{N-1} R^{i-1} \\
& \times \sum_{k=N-i+1}^{N}\left(k-\frac{1}{2}\right) \\
& +F(t) R^{N} \exp \left(\xi \frac{N^{2}}{2}\right) .
\end{aligned}
$$

Note that the first and third terms in Eq. (25) were obtained by summing the series of terms in Eq. (21). In fact, the series in the second term in Eq. (25) can also be shown to be a combination of an arithmetico-geometric series and an arithmetic series:

$$
\frac{t_{c} A \xi R}{1-R} \sum_{k=2}^{N}\left(k-\frac{1}{2}\right) R^{N-k}-\frac{t_{c} A \xi R^{N}}{1-R} \sum_{k=2}^{N}\left(k-\frac{1}{2}\right) .
$$

Both of these series can be summed over easily, so that the final equation can be written as

$$
\begin{aligned}
F(t+N \tau)= & \frac{t_{c} A\left(1-R^{N}\right)}{1-R}+\frac{t_{c} A \xi R}{1-R} S \\
& -\frac{t_{c} A \xi R^{N}}{2(1-R)}\left(N^{2}-1\right) \\
& +F(t) R^{N} \exp \left[\xi \frac{N^{2}}{2}\right],
\end{aligned}
$$

where

$$
S=\frac{N-0.5-1.5 R^{N-1}}{1-R}-\frac{R-R^{N-1}}{(1-R)^{2}}
$$

One may note that, unlike in the low-finesse case in Section 2, the last term in Eq. (27) representing the feedback is not neglected in this case. The first reason is that the terms of order $\odot\left(R^{N}\right)$ are not really negligible now because of the high value of $R$. Speaking in the language of digital filters, we can say that feedbacks are really important in this case since partial beams are stored for a long time in such cavities. The equivalent IIR filters cannot be converted into FIR ones as long as we intend to apply the linear Eq. (27) by restricting $N$ within a suitable upper limit. This limit is to be determined numerically.

The simulation procedure is the same as that described for a low-finesse cavity in Section 2 except that in step 4 there, Eq. (27) is to be used instead of Eq. (13). Since this method involves jumping a few steps of field evolution and then summing up the contribution of various partial beams, I call this method the jump-and-sump (JAS) method. It should be noted that the JAS method is not a replacement for the DFA. Rather, it is a replacement for the perturbative calculation on which the low-finesse version of the DFA is based.

The error in unit of power (i.e., the difference) made by the DFA based on the JAS method as compared with the $\mathrm{SM}$ is shown in Fig. 8(a) for $N=50$. The relative error as given by Eq. (19) is also plotted in Figs. 8(b) and 9. We see that the relative error is quite small near the resonance, but it shoots up as the field starts oscillating with higher and higher frequency. This is simply due to the fact that the frequency resolution of the method is worse than that of the exact method because of an increase by a factor of $N$ in its sampling interval. However, since the field amplitudes at those points are exceedingly small, this relative error is irrelevant for the simulation program. Because this oscillation starts decreasing soon, the relative error also decreases and soon becomes almost
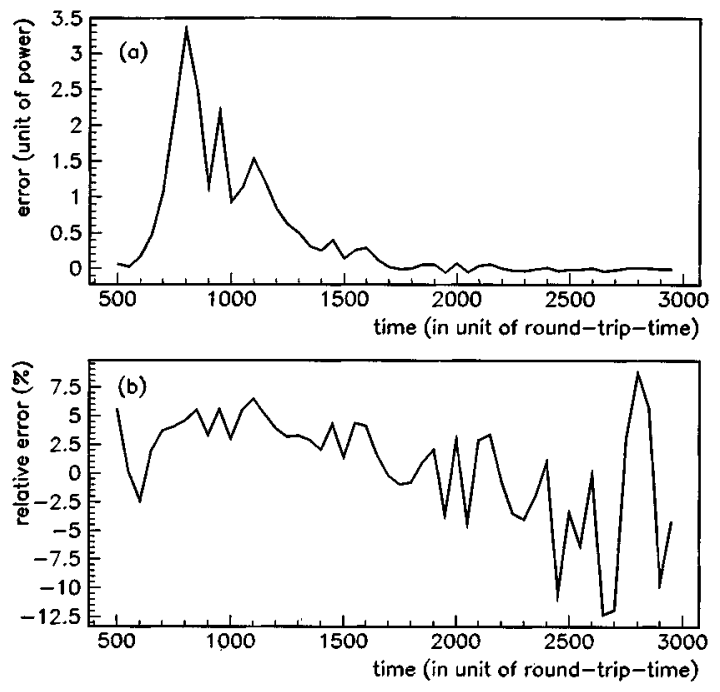

Fig. 8. (a) Error (in unit of power) and (b) relative error made by the DFA based on JAS in comparison with the SM for $N=50$ in calculating the resonance curve of Fig. 6(a). 


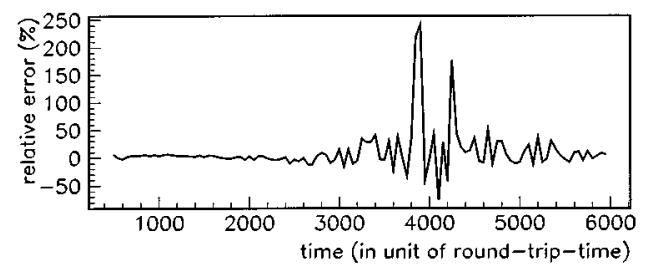

Fig. 9. Relative error (\%) made by the DFA based on JAS in comparison with the $\mathrm{SM}$ for $N=50$ in calculating the resonance curve of Fig. 6(a). Plot 8(b) is merely a zoomed-in part of this plot. The relative error increases for a short time while the field amplitude is very small; this increase is thus unimportant.

zero, much before the next resonance point arrives. (The gap between two resonance points for $w=1 \mu \mathrm{m} / \mathrm{s}$ is 26,600 round-trip times).

One may note that although relative error is a better measure than simple error, in the case of the high-finesse response of the cavities it may lead to some confusion because of its sudden increase after a resonance peak is crossed. From the next section on, only relative error in a certain important range of time around the resonance point will be plotted, as in Fig. 8(b).

Numerical investigation shows that the DFA based on JAS works quite well (with moderate values of $N$, say, 50) even for higher values of cavity finesse. As might be obvious, when applied to low-finesse cavities, this method produces the same level of error as does DFA based on perturbative calculation.

\section{SIMULATION OF THREE-MIRROR COUPLED CAVITY}

A power-recycled interferometer with a FP cavity in each arm, which is constituted by static mirrors, can be thought to be equivalent to a three-mirror cavity. Study of such a simpler geometry of the optical arrangement can thus provide us answers to some of the issues that are in common with a complete FP-type power-recycled interferometer, e.g., coupling of intracavity fields and sensitivity to misalignment. The resonance properties of such a cavity are in general quite complex; the mode losses may depend on the distribution of the optical field between the two cavities. ${ }^{10}$ The static properties of such coupled cavities have been discussed in Refs. 11 and 12. Some tabletop experiments have also been performed to study the frequency response of such a coupled cavity. ${ }^{13,14}$

In the dynamical case, however, such an equivalence between the full power-recycled interferometer with FP arm cavities and a three-mirror cavity can be established only under some simplifying assumptions, i.e., that the beam splitter is static and that the movement of the input mirror or the end mirror of one arm cavity exactly mimics the movement of the corresponding mirror in the other arm cavity (the movement mentioned in the present context is measured with respect to the beam splitter).

Alternatively, such an interferometer can be thought to be equivalent to two dynamical three-mirror-cavity systems coupled with each other through a shared part of their recycling cavities. The nature of this coupling is investigated in Section 6 . So one important step toward the final goal of understanding and simulating the dy- namical response of a power-recycled interferometer would be to investigate the physical effects and test the applicability of the numerical methods developed for twomirror cavities in a three-mirror coupled cavity.

In this section it is shown that the evolution of fields in three-mirror cavity is just like that in a two-mirror cavity whose input mirror reflectivity is changing with time. Under a general dynamical condition, most of the time this reflectivity is quite high. Using this idea, I apply the method of the DFA based on JAS introduced in the case of high-finesse cavities in Section 3 and also incorporate a special technique, called freezing the finesse, which takes care of the changing reflectivity, to write the fast DFA code for a three-mirror cavity.

\section{A. Field Equations in a Dynamical Three-Mirror Cavity}

The notation to be used for the calculation of fields in a three-mirror coupled cavity is shown in Fig. 10. The amplitude reflectivities of the input mirror (the recycling mirror for a dynamically equivalent interferometer), the middle mirror (the input mirror of arm cavities of a dynamically equivalent interferometer) and the end mirror are represented by $r_{1}, r_{2}$, and $r_{3}$, respectively. The numerical values chosen for them for the simulation to be described below are $0.96,0.94$, and 0.9999 , respectively, which are the same as those to be used for corresponding mirrors in the VIRGO interferometer. ${ }^{4}$

The exact equations for the intracavity fields in a threemirror coupled cavity when all mirrors move can now be written as follows:

$$
\begin{aligned}
B(t)= & t_{1} A \exp j\left[p_{1}(t)\right]-r_{1} r_{2} B\left(t-2 \tau_{r}\right) \\
& \times \exp j\left[\phi_{r}-p_{1}\left(t-2 \tau_{r}\right)+2 p_{2}\left(t-t_{r}\right)\right. \\
& \left.-p_{1}(t)\right]+t_{2} r_{1} r_{3} D\left(t-2 \tau_{c}-\tau_{r}\right) \\
& \times \exp j\left[\phi_{c}+\phi_{r} / 2-p_{2}\left(t-2 \tau_{c}-\tau_{r}\right)\right. \\
& \left.+2 p_{3}\left(t-\tau_{c}-\tau_{r}\right)-p_{1}(t)\right], \\
D(t)= & t_{2} B\left(t-\tau_{r}\right) \exp j\left[\frac{1}{2} \phi_{r}-p_{1}\left(t-\tau_{r}\right)+p_{2}(t)\right] \\
& +r_{2} r_{3} D\left(t-2 \tau_{c}\right) \exp j\left[\phi_{c}-p_{2}\left(t-2 \tau_{c}\right)\right. \\
& \left.+2 p_{3}\left(t-\tau_{c}\right)-p_{2}(t)\right],
\end{aligned}
$$

where $\phi_{r}$ and $\phi_{c}$ are initial (constant) round-trip phase offsets in recycling and arm cavities, respectively; $\tau_{r}$ and

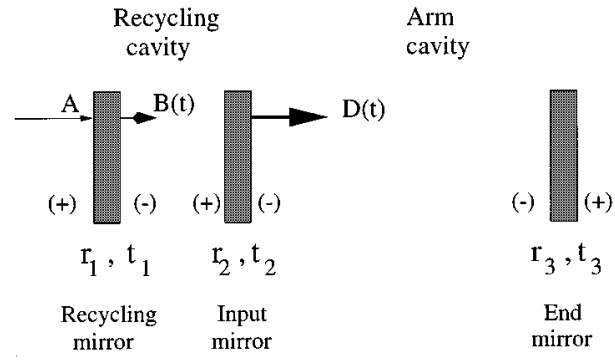

Fig. 10. Notation used for three-mirror coupled cavity; the (+) or (-) sign on two sides of a mirror indicates the phase (i.e., either zero or $\pi$ ) that a light beam acquires on reflection from that side of the mirror. 
$\tau_{c}$ are one-trip time in recycling and arm cavities, respectively; $p_{i}(t)$ are changing phase offsets (one-trip) that are due to the motion of mirror $i$, i.e., $p_{i}(t)=(2 \pi / \lambda) x_{i}, x_{i}$ representing displacement; the positive direction of motion is set to be from the first (recycling) mirror toward the third (end) mirror, and the reference point for the mirror movement is taken to be the laser source.

If we assume that the mirrors are not moving at all or are moving so slowly that their motion does not affect any field for a long time, then we can arrive at the following quasi-static expressions for $B$ and $D$ :

$$
\begin{aligned}
& B_{0}=A t_{1}\left[1-r_{2} r_{3} \exp \left(j \phi_{c}\right)\right] / \chi_{0}, \\
& D_{0}=A t_{1} t_{2} \exp \left(j \phi_{r} / 2\right) / \chi_{0},
\end{aligned}
$$

where

$$
\begin{aligned}
\chi_{0}=1 & +r_{1} r_{2} \exp \left(j \phi_{r}\right)-r_{2} r_{3} \exp \left(j \phi_{c}\right) \\
& -r_{1} r_{3}\left(r_{2}^{2}+t_{2}^{2}\right) \exp \left(j \phi_{r}+j \phi_{c}\right) .
\end{aligned}
$$

The double-resonance condition is achieved when $\phi_{c}$ $=2 n \pi$ and $\phi_{r}=2 m \pi$, where $n$ and $m$ are integers. The numerical values for $\left|B_{0}\right|^{2}$ and $\left|D_{0}\right|^{2}$ in the doubleresonance condition are $\approx 42 \mathrm{~W}$ and $\approx 1350 \mathrm{~W}$, respectively, for $|A|^{2}=1.0 \mathrm{~W}$, and the values of reflectivities are as chosen above for VIRGO. In the static case, the power transmitted through the system is maximum at this point. ${ }^{11}$ However, in general, a maximum point of transmission may occur at a value $\phi_{c} \neq 2 n \pi$ for a value of $\phi_{r} \neq 2 m \pi$. The rules of occurrence of these resonance peaks will be discussed in Section 5 for both static and dynamical cases.

The exact numerical code for the three-mirror cavity is written on the basis of Eqs. (29) and (30), as shown in Fig. 11. It should be noted that this code really is exact only if the ratio of the lengths of the arm cavity and the recycling cavity is an integer $\rho$. It is not however, a very important consideration, because, in the case when $\rho$ is not an integer, a code like this based on the nearest integer value of $\rho$ would make only a negligible error with respect to a code that takes into account the real value of $\rho$. The reason is that in almost all cases of interest, we need not study the system with a very fine level of frequency resolution (i.e., with a sampling period smaller than the round-trip time in recycling cavity).

The exact dynamical double-resonance curve for the field $B$, where only the end mirror moves with a velocity of $1 \mu \mathrm{m} / \mathrm{s}$ and $\phi_{r}$ is set to a value of $2 m \pi$, is shown in Fig. 12. It so turns out that the corresponding resonance curve for the field $D$ looks exactly like what was plotted in Fig. 6(a) for a two-mirror high-finesse cavity with $r_{c}$ $=0.998733$, whose length was changing at the same rate. The following discussion explains this.

The exact code as represented in Fig. 11 takes a very long time to compute the field evolution. Therefore we need to develop a faster way of doing this by finding some ways of writing nonexact equations for three-mirror coupled cavities. One way of doing this is to replace the recycling cavity by an equivalent mirror whose reflectivity $r_{\text {rec }}$ depends on the dynamical phase offset denoted by $\theta_{r}$ in the recycling cavity and then to write down the

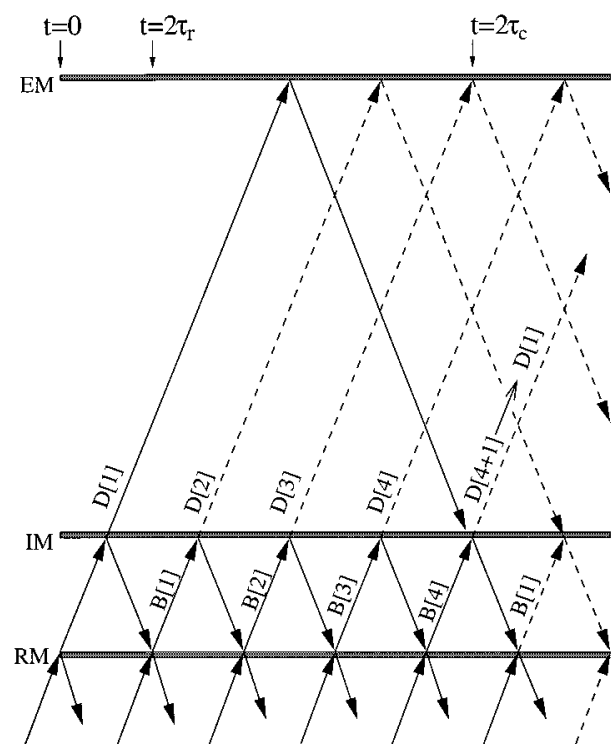

Fig. 11. Exact dynamical code written for a three-mirror cavity for which the ratio of lengths of the arm cavity and the recycling cavity is an integer, $\rho$. The figure shows the case for $\rho=4$; For the equivalent case of the VIRGO interferometer, $\rho$ has been assumed to be $250(=3000 / 12)$. Note that at any moment of time the code needs to memorize $2 \rho$ number of data for the evolution of phases in two cavities and $\rho$ number of past values for the field D. RM, recycling mirror; IM, input mirror; EM, end mirror.

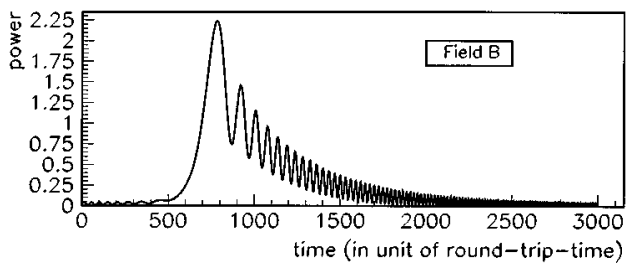

Fig. 12. Exact dynamical double-resonance curve for the field $B$ in a three-mirror cavity while only the end mirror moves with a velocity $1 \mu \mathrm{m} / \mathrm{s}$ and the round-trip phase in the recycling cavity $\theta_{r}$ is set to an integral multiple of $2 \pi$. Input power is one unit.

equation for $D$ in the resulting two-mirror cavity constituted by the end mirror and the equivalent mirror for the recycling cavity:

$$
D(t)=t_{\text {rec }}(t) A+r_{\text {rec }}(t) r_{3} \exp \left[j \theta_{c}\left(t-\tau_{c}\right)\right] D\left(t-2 \tau_{c}\right),
$$

where

$$
\begin{aligned}
& r_{\mathrm{rec}}(t)=\frac{r_{2}+r_{1}\left(r_{2}^{2}+t_{2}^{2}\right) \exp \left[j \theta_{r}(t)\right]}{1+r_{1} r_{2} \exp \left[j \theta_{r}(t)\right]}, \\
& t_{\mathrm{rec}}(t)=\frac{t_{1} t_{2} \exp j\left[\theta_{r}(t) / 2+p_{1}(t)\right]}{1+r_{1} r_{2} \exp \left[j \theta_{r}(t)\right]} .
\end{aligned}
$$

The field $B$ can then be easily calculated with this equation. The simulation program based on these equations now runs approximately 270 times faster, because the time-sampling rate is much smaller now (i.e., $1 / \tau_{c}$ instead of $1 / \tau_{r}$ ); also it can perform much less expensive algebraic calculations without memorizing any data in a buffer.

We find that this equation makes negligible (in fact, zero up to several decimal points near the peak) relative error compared with the exact code. However, in this 
case also, the relative error increases rapidly for some time after the resonance peak is crossed, as the fields start oscillating with higher and higher frequencies. This is due to the reduced frequency resolution of the method, which effectively increases the sampling period by a factor of $\rho$. As already explained in Section 3 for the case of the DFA based on JAS, this error is unimportant since the absolute value of the field is very low in that range. Obviously, this level increases as the ratio of lengths of the arm cavity and the recycling cavity decreases and vice versa.

The reason that Eq. (34) can perform such accurate calculation of fields is as follows. The missing information in the equation, $\tau_{r}$, is only $40 \mathrm{~ns}$ compared with $\tau_{c}$, which is $\sim 10 \mu \mathrm{s}$. So any extra information that $\tau_{r}$ could have contributed to the dynamical response amounts to a negligible time gap between cause and effect compared with the evolution of fields during the one-trip time in the arm cavity. For the same reason, if we had tried the other way round by replacing the arm cavity with an equivalent mirror, we would have written completely wrong equations for the dynamical evolution of fields.

On the basis of the above observation, we may make an assumption that $\tau_{r}$ can be neglected with respect to $\tau_{c}$ in the exact set of equations. This approximation was first used by Redding ${ }^{15}$ and has also been applied by others ${ }^{9,16}$ to study the response of a three-mirror cavity. From now on, I refer to this as a small-recycling-cavity (small-reccav) approximation.

Using this approximation, the full dynamical equations (29) and (30) can now be written as

$$
\begin{aligned}
B(t+\tau)= & \frac{1}{\chi(t+\tau)}\left\{t_{1} A \exp j\left[p_{1}(t+\tau)\right]\right. \\
& +t_{2} r_{1} r_{3} D(t) \exp j\left[\frac{\theta_{r}(t+\tau)}{2}\right. \\
& \left.\left.+\theta_{c}(t+\tau / 2)\right]\right\}, \\
D(t+\tau)= & t_{\text {rec }}(t+\tau) A+r_{\text {rec }}(t+\tau) r_{3} D(t) \\
& \times \exp j\left[\theta_{c}(t+\tau / 2)\right],
\end{aligned}
$$

where $\tau=2 \tau_{c}$ and

$$
\begin{aligned}
t_{\mathrm{rec}}(t) & =\frac{1}{\chi(t)} t_{1} t_{2} \exp j\left[\frac{1}{2} \theta_{r}(t)+p_{1}(t)\right] \\
r_{\mathrm{rec}}(t) & =r_{2}+\frac{t_{2}^{2} r_{1} \exp j\left[\theta_{r}(t)\right]}{\chi(t)} \\
\theta_{r}(t) & =\phi_{r}-2 p_{1}(t)+2 p_{2}(t) \\
\theta_{c}\left(t-\tau_{c}\right) & =\phi_{c}-p_{2}\left(t-2 \tau_{c}\right)+2 p_{3}\left(t-\tau_{c}\right)-p_{2}(t) \\
\chi(t) & =1+r_{1} r_{2} \exp j\left[\theta_{r}(t)\right]
\end{aligned}
$$

\section{B. Simulation by Jump and Sump While Freezing the Finesse}

We may now ask ourselves if it would be possible to compute even faster than the short-rec-cav approximation allows. To answer this question, we note that the evolu-

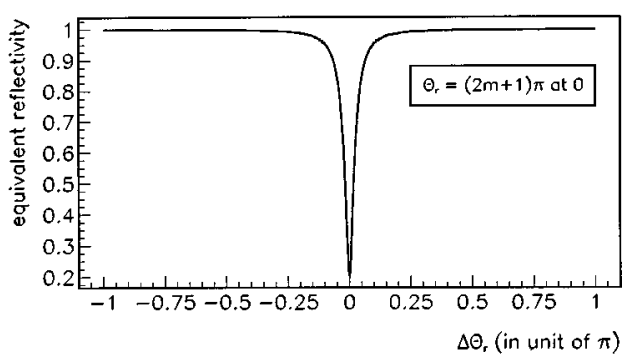

Fig. 13. Equivalent amplitude reflectivity of the recycling mirror, $r_{\text {rec }}$, is plotted as a function of round-trip phase offset $\Delta \theta_{r}$ in the recycling cavity.

tion of field $D$ as represented by Eq. (38) is just like that of the intracavity field $F$ of a two-mirror cavity whose input-mirror reflectivity $r_{c} \equiv r_{\text {rec }}$ is changing with time:

$F(t+\tau)=t_{c}(t+\tau) A+F(t) R(t+\tau) \exp [j \theta(t+\tau / 2)]$,

where $R(t)=r_{e} r_{c}(t) \exp \left(j \phi_{i}\right)$ is the product of the amplitude reflectivities of the end mirror $r_{e}$, the input mirror $r_{c}$, and a very small constant phase offset $\phi_{i}$ in a roundtrip of the cavity when mirrors are not moving. The evolution of field $B$ represented by Eq. (37) is completely dependent on that of $D$, which means that if we can compute $D$ faster, we can compute $B$ also at the same speed. Let us plot the reflectivity $r_{\text {rec }}$ as a function of $\Delta \theta_{r}=\theta_{r}-(2 m+1) \pi$ in Fig. 13. One can see that except for values very near $(2 m+1) \pi$ for $\theta_{r}$, the value of $r_{\text {rec }}$ is always quite high and very near $\approx 0.998733$, the value at $\theta_{r}=2 m \pi$. This explains why the dynamical double-resonance curve for the field $D$ in a three-mirror cavity has a behavior exactly like that of a high-finesse two-mirror cavity with $r_{c}=0.998733$ [as in Fig. 6(a)], when we assume that only the end mirror is moving and set $\theta_{r}=2 m \pi$.

So we now have a ready answer to the above question. If we consider the simplest case of constant $r_{\text {rec }}$ by assuming "only end mirror moving," we just need to apply the DFA based on JAS to Eqs. (38) to develop a code that is much faster than what the small-rec-cav approximation can ensure. For example, the error level for JAS compared with the equations approximated by small-rec-cav for the field $D$ in this simple case, when we allow only the end mirror to move and set $\theta_{r}=2 m \pi$, is same as that shown in Fig. 8.

However, to have a complete simulation, we need also to consider the dynamics of the recycling cavity and thus the changing values of $r_{\text {rec }}$. By comparing Eq. (44) with Eq. (38), we note that in the equivalent expressions for $r_{c}(t)$ and $t_{c}(t)$, i.e., $r_{\text {rec }}$ and $t_{\text {rec }}$, respectively, the time dependence arises only through the dependence of $\theta_{r}$ on time. We noted that except for a small range of values of $\theta_{r}$ near $2 m \pi$, the equivalent reflectivity $r_{\text {rec }}(t)$ does not change much from unity. This leads me to incorporate a special technique that I call freezing the finesse ${ }^{17}$ (FTF). As the name suggests and as is explained below, I set the values of $\theta_{r}$ and so of $r_{c}$ and $t_{c}$ to suitable constants before each step of the JAS method, thus effectively freezing the equivalent finesse of the three-mirror cavity during each interval of jumping. 
So, the procedure of simulation for three-mirror cavities when all mirrors move is as follows:

- Step 1: Time is sliced into equal intervals of width $\Delta=N \tau$, so that any time $t_{i}=i N \tau$, where $i$ is an integer.

- Step 2: If we assume that for the short time interval $\left(t_{i}, t_{i+1}\right)$ the mirrors move with constant velocities, then by defining the rates of change of the phase offsets in the recycling and the arm cavity, respectively, as

$$
w_{r}=v_{2}-v_{1}, \quad w_{c}=v_{3}-v_{2},
$$

we can write

$$
\theta_{r}(t)=\phi_{r}+\frac{4 \pi}{\lambda} w_{r} t, \quad \theta_{c}(t)=\phi_{c}+\frac{4 \pi}{\lambda} w_{c}\left(t-\tau_{c}\right) .
$$

- Step 3 (FTF): The phase $\phi_{i}$ and the reflectivity $r_{c}$ in the expression for $R$ and $t_{c}$ in Eq. (44) are fixed to the following values during any time interval $\left(t_{i}, t_{i+1}\right)$ :

$$
\begin{aligned}
\phi_{i} & =\theta_{c}\left(t_{i}\right), \\
r_{c i} & =\frac{1}{2}\left[r_{\mathrm{rec}}\left(t_{i}\right)+r_{\mathrm{rec}}\left(t_{i+1}\right)\right], \\
t_{c i} & =t_{\mathrm{rec}}\left(t_{i+1}\right) .
\end{aligned}
$$

- Step 4 (JAS): The field at $t_{i+1}, D(t+N \tau)$ is obtained from the value of the field at $t_{i}, D(t)$ by simply substituting $D \rightarrow F, r_{3} \rightarrow r_{e}, r_{c i} \rightarrow r_{c}, t_{c i} \rightarrow t_{c}$, and $w_{c} \rightarrow w$ in Eq. (27).

- Step 5: The values of $\phi_{i+1}$ and $w_{c}$ are changed, and $D_{i+2}$ is calculated.

Before we discuss the error levels of such a simulation, we note that if $p_{1}$ were a constant, that would not have affected the evolution of intracavity field amplitudes in any way. The variation in $p_{1}$, i.e., the velocity $v_{1}$, however, affects the field amplitudes whenever the operating condition of the beam corresponds to a high value of effective finesse (i.e., whenever $r_{\text {rec }}$ is high) for the threemirror system. So if we completely neglect $p_{1}$ in Eqs. (37) and (38), it leads to almost no error if the beam is on exact antiresonance in the arm cavity and on resonance in the recycling cavity [i.e., $\theta_{c}=(2 n+1) \pi$ and $\theta_{r}=(2 m$ + 1) $\pi$; effective finesse is the lowest]. However, it leads to the same level of error as shown in Fig. 7 when the beam is on or near double resonance (i.e., $\theta_{c}=2 n \pi$ and $\theta_{r}=2 m \pi$; effective finesse is the highest). Throughout the following discussion on error levels, we neglect this phase factor arising out of the Doppler shift of the incoming light with respect to the source.

As we can expect, the error made by the technique of FTF depends strongly on the first derivative of $r_{\text {rec }}$ with respect to time. So we can guess that the error will be less as long as $r_{\text {rec }}$ varies slowly, which it does almost throughout the range of $\theta_{r}$ except for a small range (i.e., about $\pm 0.2 \pi)$ near $\theta_{r}=\pi$.

In Fig. 14(a) I plot a double-resonance curve for the field $D$, using Eq. (38), while phases change with rates: $w_{r}=w_{c}=1 \mu \mathrm{m} / \mathrm{s}$. The same resonance curve, when drawn by using the faster DFA based on JAS and FTF for $N=30$, gives relative error with respect to the equations based on small-rec-cavity, as shown in Fig. 14(b). As has been explained, the error is quite small near resonance and increases for some time when the field oscillates rap-
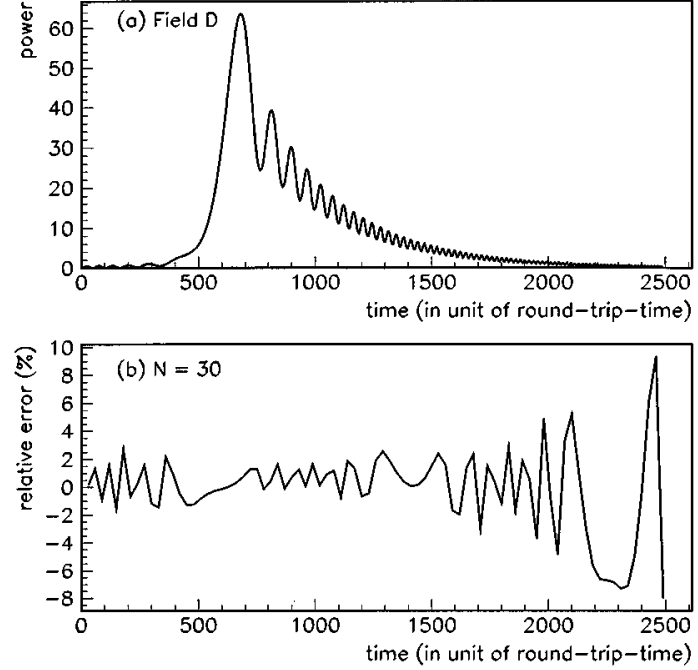

Fig. 14. (a) Dynamical double-resonance curve for field $D$ in a three-mirror cavity drawn by using equations based on only the small-rec-cav assumption for $w_{r}=w_{c}=1 \mu \mathrm{m} / \mathrm{s}$. Input power is one unit. (b) Relative error by the DFA based on JAS and FTF with respect to only small-rec-cav in calculating the same curve for $N=30$. The relative error increases for a short time while the field amplitude is very small; this increase is thus unimportant.
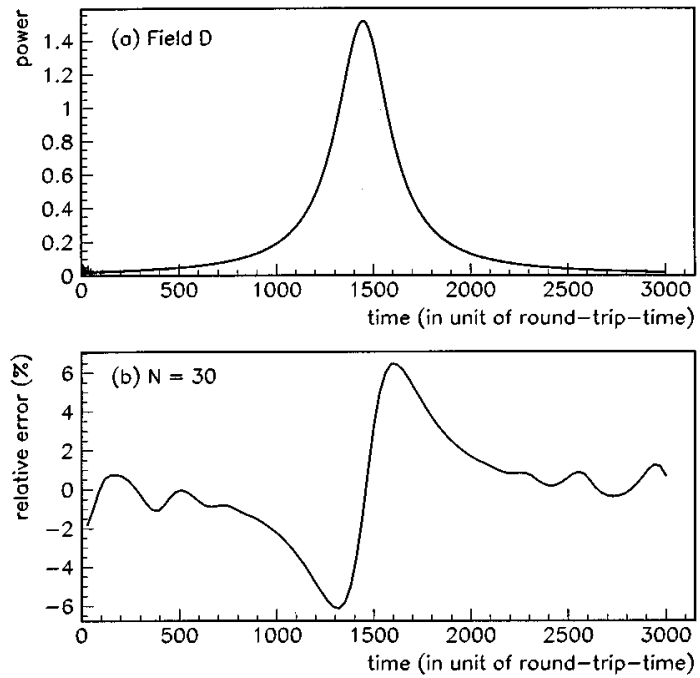

Fig. 15. (a) Resonance peak of field $D$ in a three-mirror cavity when the beam is on exact antiresonance in the arm and on resonance in the recycling cavity, as drawn by equations based on only the small-rec-cav assumption for $w_{r}=w_{c}=1 \mu \mathrm{m} / \mathrm{s}$. Input power is one unit. (b) Relative error by the DFA based on JAS and FTF with respect to only small-rec-cav in calculating the same curve for $N=30$.

idly with very small amplitude. For $N=40$ the error near resonance varies between $\pm 3 \%$, and for $N=50$ the figure increases to about $\pm 5 \%$. For other combinations of $w_{r}$ and $w_{c}$ the error levels vary, but in all cases for same $N$, these continue to have the same order of magnitude, provided that the velocities do not become too high in comparison with $1 \mu \mathrm{m} / \mathrm{s}$.

Now we discuss the error consideration when the effective finesse of the system is near the lowest, i.e., when the beam is on exact antiresonance $\left[\theta_{c}=(2 n+1) \pi\right]$ in the arm cavity but on resonance $\left[\theta_{r}=(2 m+1) \pi\right]$ in the re- 
cycling cavity, while the phases change as $w_{r}=w_{c}$ $=1 \mu \mathrm{m} / \mathrm{s}$. The peak for this condition is drawn in Fig. 15(a) by using Eq. (38). The relative error in drawing the same curve by using the DFA based on JAS and FTF for $N=30$ with respect to the curve in Fig. 15(a) is plotted in Fig. 15(b). As expected, the error now is more than in the case of double resonance. The peak of the error increases to $\sim 8 \%$ for $N=40$ and $\sim 10 \%$ for $N=50$. For other combinations of $w_{r}$ and $w_{c}$, unlike in the previous case, the error levels do not change much, owing to lower values of effective finesse in this region.

So we see that for any value of $N$, the simulation error is the least for a beam on double resonance. When the operating condition of the beam is anywhere else, the error in simulation increases. When the operating condition gets to the exact antiresonance point in the arm cavity but to the individual resonance point in the recycling cavity, the simulation for the DFA based on JAS and FTF runs with its highest level of error. Even the highest level of error is quite tolerable for (say) $N=50$ for which the program runs at a speed $\sim 30$ times faster than a code based only on the approximation of small-rec-cav [Eqs. (37) and (38)].

\section{THREE-MIRROR COUPLED CAVITY: WHAT HAPPENS WHEN ALL MIRRORS MOVE}

Now that we have a fast simulation code in hand, we can use it to perform some experiments ${ }^{18}$ with the aim of obtaining some physical insights into the coupling of the intracavity fields in a three-mirror cavity. Specifically, I study how and when the peaks for the intracavity fields of a three-mirror cavity appear in both static and dynamical cases. The study of peaks will also be of use for writing an algorithm for starting up a power-recycled interferometer to bring all its constituent cavities near the operating condition.

In Subsection 5.A I describe how various curves are drawn to bring out physical issues related to the field distribution in the two cavities and tabulate some values after introducing various important parameters. In Subsection 5.B I discuss all these physical points. It should be noted here that although these discussions address three-mirror coupled cavities, the conclusions drawn here are, however, very important and are of great use when we start discussing the full power-recycled interferometer.

\section{A. Dynamical Curves and the Corresponding Quasi- Static Curves}

\section{Dynamical Curves}

As shown in Eqs. (37) and (38), intracavity fields are functions of two parameters, the round-trip phase offsets $\theta_{r}$ and $\theta_{c}$. However, if we assume constancy of velocities of mirrors for a short time, we can express these phases in the unit of those acquired as a result of mirror motion in one round-trip time (rtt) of the arm cavity. Such a unit provides an advantage in terms of plotting the power of the fields as a function of only one parameter, the number of round-trips, instead of two, $\theta_{r}$ and $\theta_{c}$, as shown in Figs.
16-23. So phases acquired in one $\mathrm{rtt}$ by $\theta_{r}$ and $\theta_{c}$ are given by $K_{r} \zeta$ and $K_{c} \zeta$, respectively, where $\zeta$ is the number of rtt's and so

$$
\begin{array}{ll}
\theta_{r}(\zeta)=K_{r} \zeta+\phi_{r}, & K_{r}=\left(\frac{2 \pi}{\lambda}\right) 2 \tau w_{r} \\
\theta_{c}(\zeta)=K_{c} \zeta+\phi_{c}, & K_{c}=\left(\frac{2 \pi}{\lambda}\right) 2 \tau w_{c} .
\end{array}
$$

\section{How to Follow Figs. 16-23}

Figures 16-23 are generated for various cases pertaining to dynamical conditions of a three-mirror cavity. In all of these figures, the rates of change of the lengths of the recycling and arm cavities, $w_{r}$ and $w_{c}$, have been chosen to be 1.7 and $1.0 \mu \mathrm{m} / \mathrm{s}$, respectively, and the input power is assumed to be 1.0 unit. Notice that different values for velocities have been chosen to keep the discussion at a general level. Assumption of the same rates of change might have made our discussion much simpler but at the cost of generality and reality.
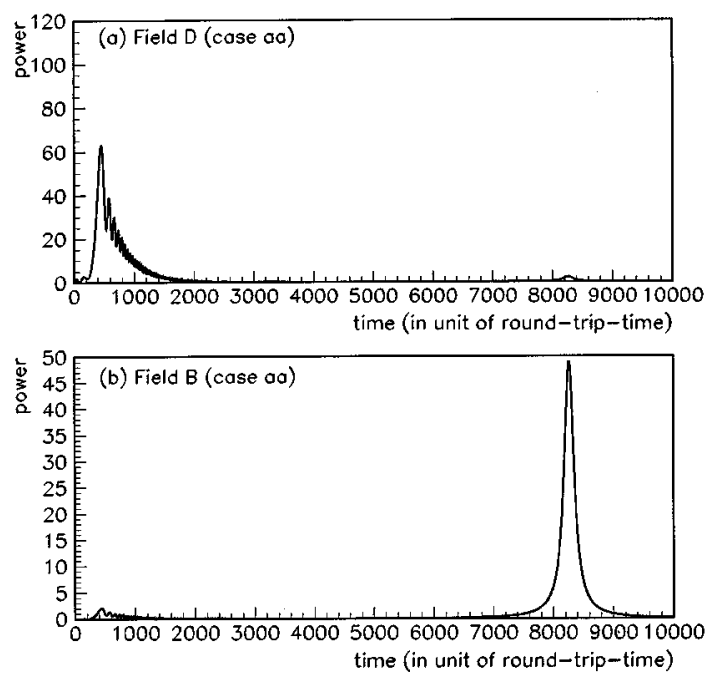

Fig. 16. Evolution of fields (a) $D$ and (b) $B$ in case aa, which corresponds to the double-resonance condition for first peaks.
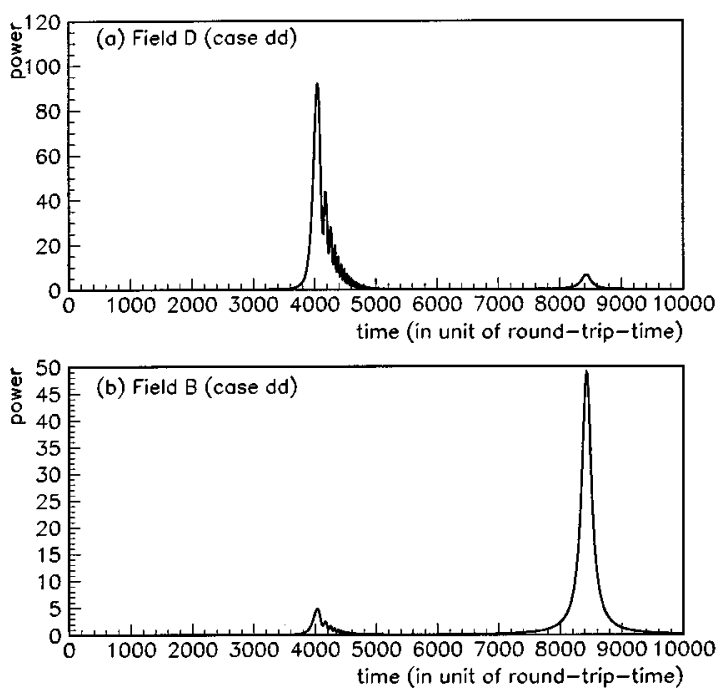

Fig. 17. Evolution of fields (a) $D$ and (b) $B$ in case dd. 

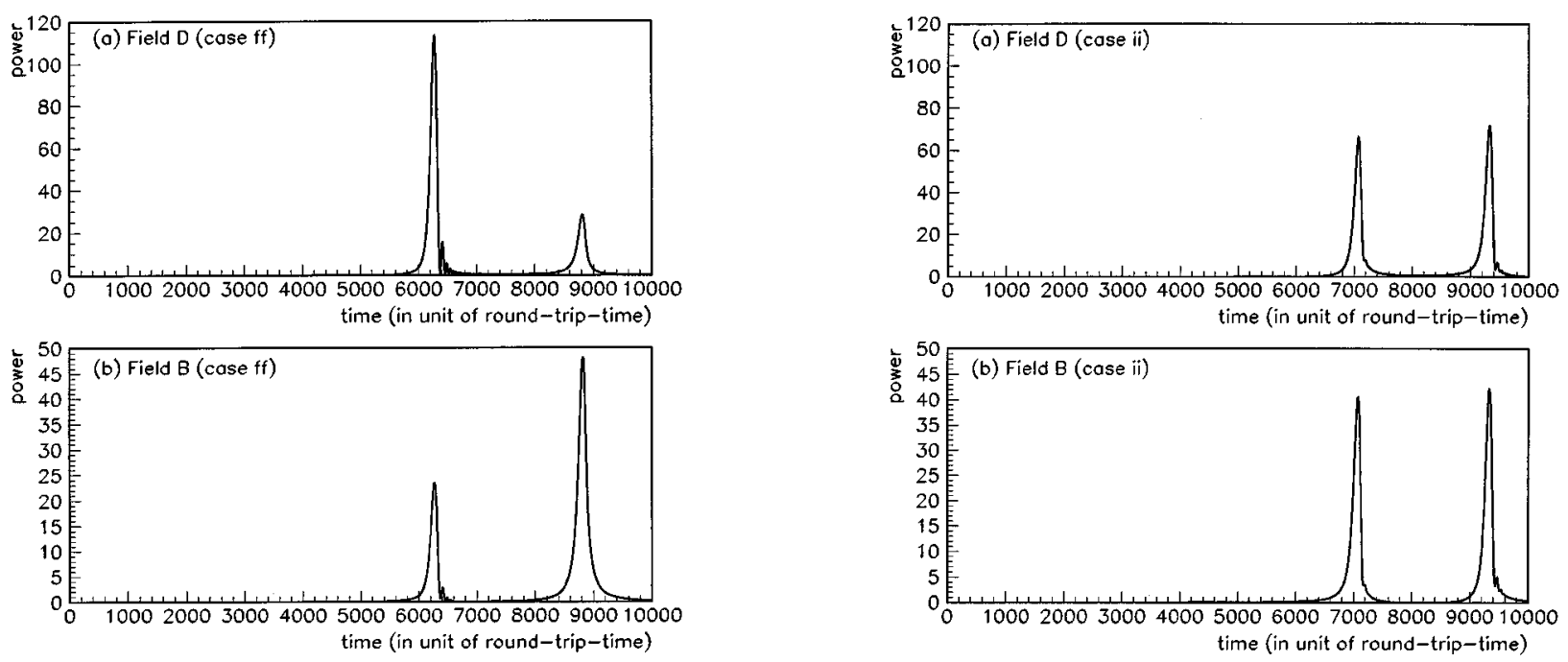

Fig. 18. Evolution of fields (a) $D$ and (b) $B$ in case ff.
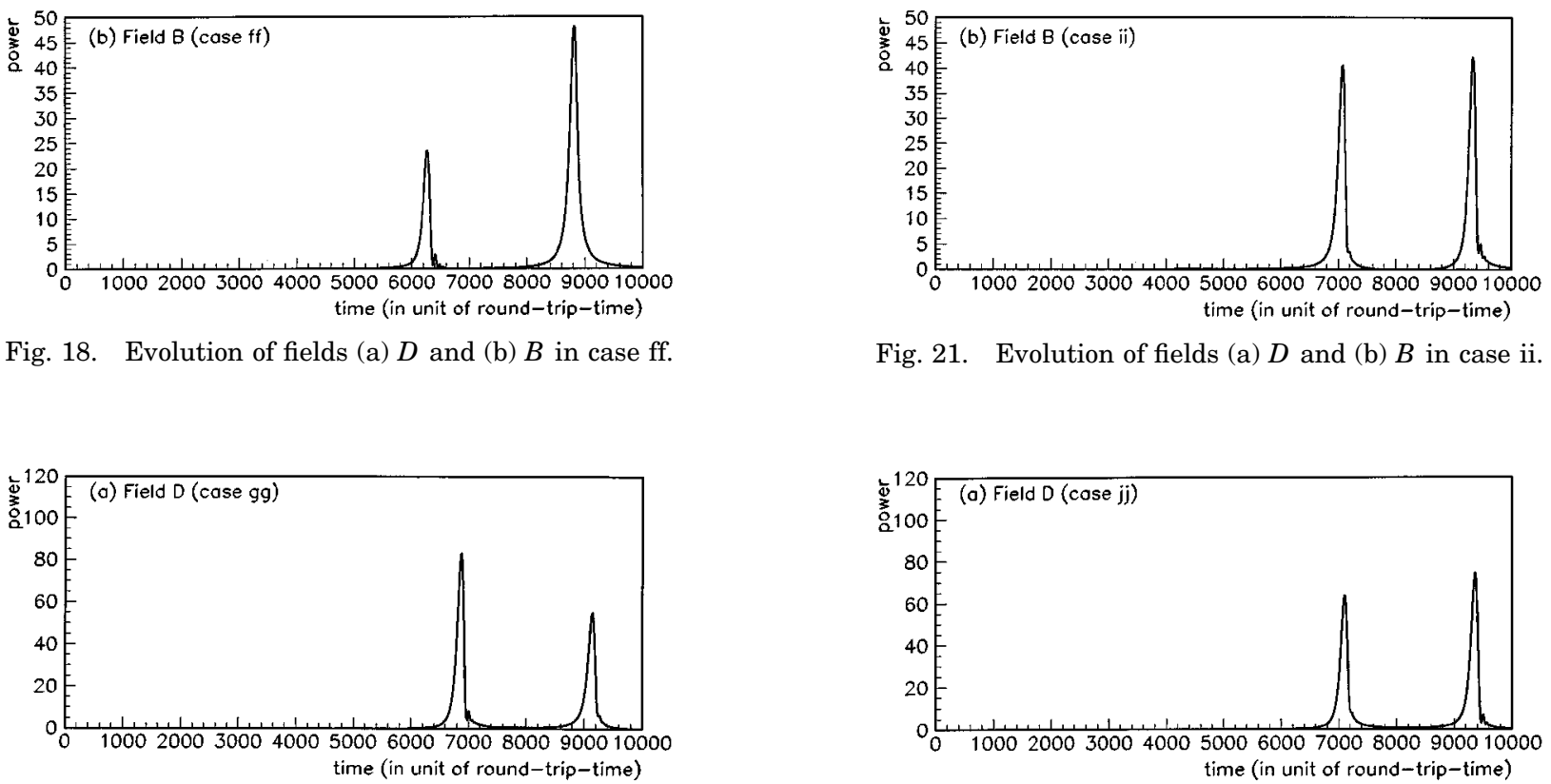

Fig. 21. Evolution of fields (a) $D$ and (b) $B$ in case ii.
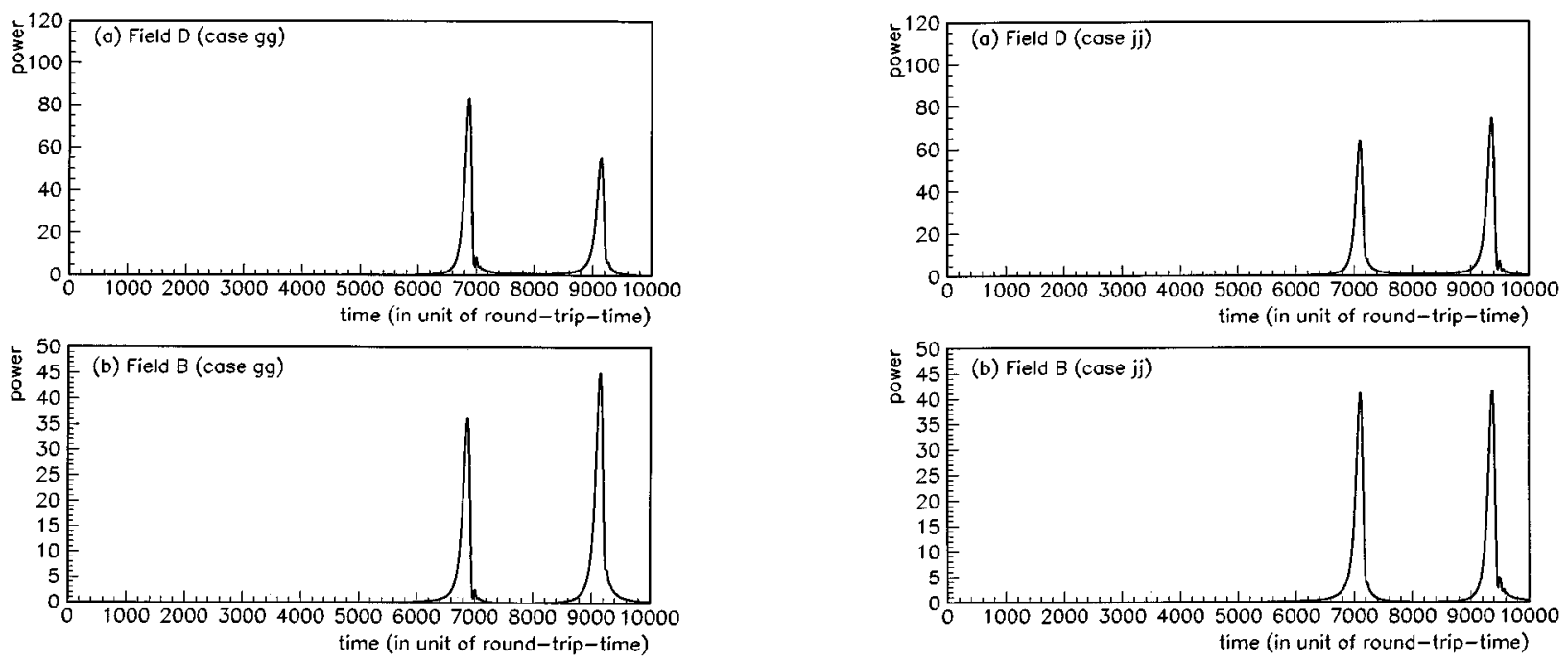

Fig. 19. Evolution of fields (a) $D$ and (b) $B$ in case gg.

Fig. 22. Evolution of fields (a) $D$ and (b) $B$ in case jj.
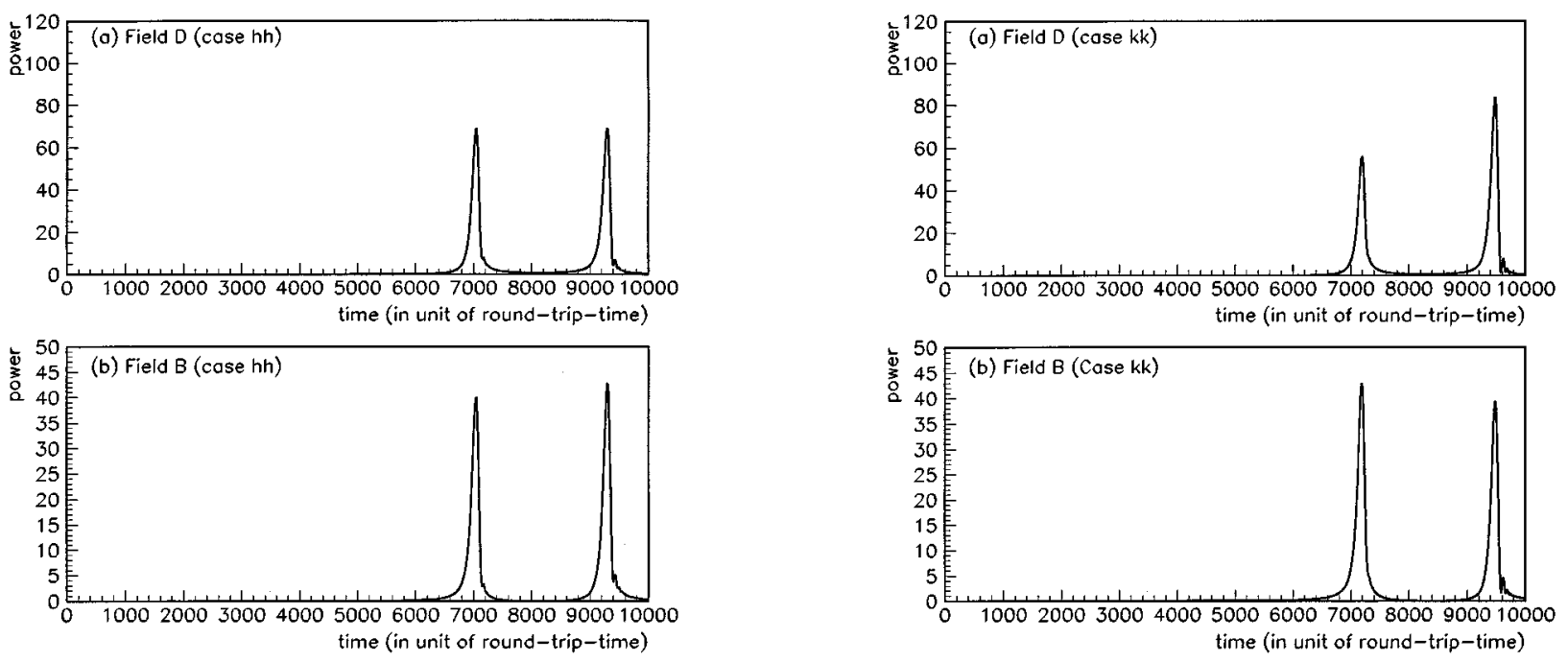

Fig. 20. Evolution of fields (a) $D$ and (b) $B$ in case hh.

Fig. 23. Evolution of fields (a) $D$ and (b) $B$ in case kk. 
Table 2. Numerical Values of Some Important Quantities for the Dynamical Simulation of Three-Mirror Coupled Cavities under Different Conditions

\begin{tabular}{|c|c|c|c|c|c|c|}
\hline Case & $\zeta_{0}$ & $\nabla$ & $P_{1}(\mathrm{DY})$ & $\zeta_{1}(\mathrm{DY})$ & $P_{2}(\mathrm{DY})$ & $\zeta_{2}(\mathrm{DY})$ \\
\hline aa* & 324 & $+0.588235 \pi$ & 63 & 450 & 2.3 & 8262 \\
\hline $\mathrm{bb}$ & 1497 & $+0.5 \pi$ & 66 & 1565 & 3 & 8298 \\
\hline $\mathrm{cc}$ & 2827 & $+0.4 \pi$ & 76 & 2817 & 4.2 & 8351 \\
\hline $\mathrm{dd}^{*}$ & 4157 & $+0.3 \pi$ & 90 & 4046 & 6.5 & 8430 \\
\hline ee & 5487 & $+0.2 \pi$ & 114 & 5222 & 13 & 8563 \\
\hline $\mathrm{ff}^{*}$ & 6817 & $+0.1 \pi$ & 115 & 6278 & 28 & 8815 \\
\hline gg* & 7748 & $+0.03 \pi$ & 83 & 6870 & 55.1 & 9139 \\
\hline $\mathrm{hh}^{*}$ & 8083 & $+0.0048 \pi$ & 68.8 & 7042 & 68.8 & 9297 \\
\hline ii $^{*}$ & 8147 & +0.0 & 65 & 7073 & 72 & 9330 \\
\hline $\mathrm{jj}^{*}$ & 8211 & $-0.0048 \pi$ & 63.5 & 7102 & 74.5 & 9363 \\
\hline $\mathrm{kk}^{*}$ & 8419 & $-0.0204 \pi$ & 55.4 & 7192 & 83.5 & 9480 \\
\hline
\end{tabular}

${ }^{*}$ Cases aa, dd, ff, gg, hh, ii, jj, and kk correspond to Figs. 16, 17, 18, 19, 20, 21, 22, and 23, respectively.

Table 3. Numerical Values of Some Important Quantities for the Quasi-Static Curves of Three-Mirror Coupled Cavities under Different Conditions

\begin{tabular}{|c|c|c|c|c|c|}
\hline Case & $\zeta_{0}$ & $P_{1}(\mathrm{QS})$ & $\zeta_{1}(\mathrm{QS})$ & $P_{2}(\mathrm{QS})$ & $\zeta_{2}(\mathrm{QS})$ \\
\hline aa & 324 & 1350 & 324 & 2.3 & 8260 \\
\hline $\mathrm{bb}$ & 1497 & 1290 & 1438 & 3 & 8295 \\
\hline cc & 2827 & 1100 & 2693 & 4.2 & 8347 \\
\hline dd & 4157 & 810 & 3927 & 6.5 & 8424 \\
\hline ee & 5487 & 500 & 5113 & 12 & 8550 \\
\hline $\mathrm{ff}$ & 6817 & 230 & 6188 & 28 & 8790 \\
\hline gg & 7748 & 114 & 6807 & 58 & 9092 \\
\hline hh & 8083 & 85.4 & 6988 & 76.8 & 9242 \\
\hline ii & 8147 & 81 & 7020 & 81 & 9274 \\
\hline $\mathrm{jj}$ & 8211 & 76.7 & 7052 & 85.3 & 9306 \\
\hline $\mathrm{kk}$ & 8419 & 64.4 & 7148 & 102 & 9417 \\
\hline
\end{tabular}

Throughout the discussion, $\theta_{r}$ and $\theta_{c}$ are expressed in terms of the nearest integer values of rtt. The small fractional difference between the actual (real) and the quoted (integer) value does not affect the main physical points that will be described here.

Note: In all of these figures, the points $\theta_{r}=2 m \pi(m$ is any integer) and $\theta_{r}=(2 m+1) \pi$ have been fixed at points $\zeta=324$ and $\zeta=8147$ respectively $\left(\theta_{r}\right.$ acquires a phase of $\pi$ in $8147-324=7823 \mathrm{rtt}$, whereas $\theta_{c}$ acquires the same in $13,300 \mathrm{rtt}$, since the chosen value of $w_{r}$ is greater than that of $w_{c}$ ).

In Fig. 16 the phase offset in the arm cavity is chosen to be such that the $\theta_{c}=2 \pi n$ ( $n$ is any integer) point coincides with $\theta_{r}=2 m \pi$ at $\zeta=324$, thus generating the double-resonance curve (dynamical). In the same figure, where $\theta_{r}$ is equal to $(2 m+1) \pi$ (at $\left.\zeta=8147\right)$, the value of $\theta_{c}$ is $+0.588235 \pi$, and so this corresponds to an individual resonance point of the recycling cavity, and some light leaks into the arm cavity. In the next successive figures (17-23) the initial phase offset in the arm cavity is changed in such a way that the $\theta_{c}=2 \pi n$ point gets shifted toward $\theta_{r}=(2 m+1) \pi$ before becoming coincident with it in Fig. 21 (Case ii) and then crosses the point to the other side.

Some of the interesting numerical values for these figures as well as those corresponding to the intermediate
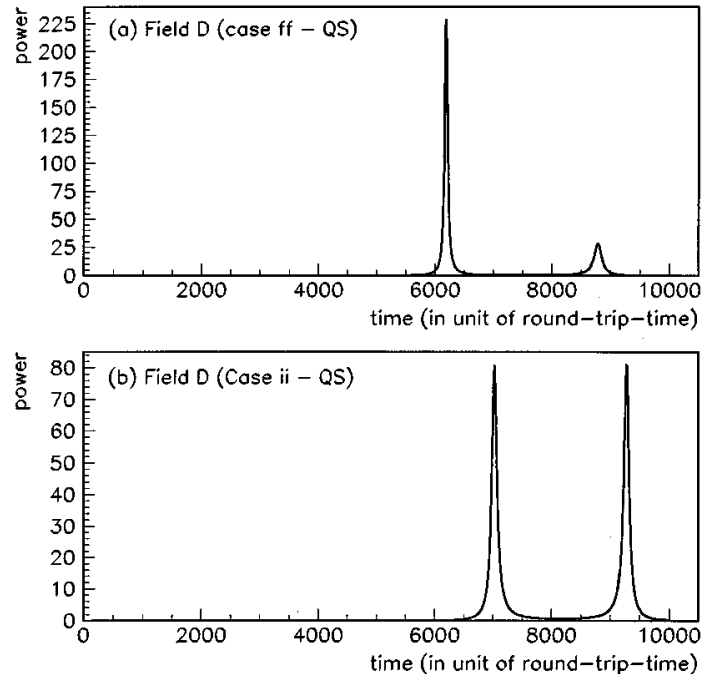

Fig. 24. Quasi-static curves for field $D$ for (a) case ff and (b) case ii.

situations are shown in Table 2. There are two peaks in each figure. The quantities, $P_{\mathrm{i}}(\mathrm{DY})$ and $\zeta_{\mathrm{i}}(\mathrm{DY})$ represent the power and location of the $i$ th peak $(i=1,2)$ for the field $D$, whereas $\zeta_{0}$ represents location of the point 
$\theta_{c}=2 n \pi$. The value of $\theta_{c}$ when $\theta_{r}=(2 m+1) \pi$ (at $\zeta$ $=8147)$ is represented by $\nabla$.

The figures are different from each other just because the $\theta_{c}=2 n \pi$ point appears at a different location in each case. So each of these figures can also be represented by the values of $\nabla$ and/or $\zeta_{0}$.

\section{Quasi-Static Curves for the Corresponding Dynamical Cases}

For a closer study of the physical nature of these curves, here I introduce what I call a quasi-static curve corresponding to each of the dynamical cases described above. In the quasi-static expressions for the intracavity fields in Eqs. (31) and (32), phases $\theta_{r}$ and $\theta_{c}$ are expressed in terms of the parameter $\zeta$ and thus in units of phases that could be acquired in a dynamical case in one rtt with $w_{r}$ $=1.7 \mu \mathrm{m} / \mathrm{s}$ and $w_{c}=1.0 \mu \mathrm{m} / \mathrm{s}$, respectively. Then the power of each of these fields is plotted as a function of $\zeta$.

For example, the quasi-static curve for field $D$ corresponding to the dynamical cases ff and ii (see Table 2) are plotted in Figs. 24(a) and 24(b), respectively. Table 3 gives numerical values for some important quantities for the quasi-static curves corresponding to the dynamical cases described in Table 2. In quasi-static curves, we plot something static in a seemingly dynamical way. These curves just represent how the intracavity fields in a static three-mirror cavity vary if these are measured step by step by changing the phase offsets in the two cavities at each step in such a way that these changes mimic the corresponding dynamical case in a discrete fashion. So these curves provide us the actual values of the phases for the resonance points, i.e., a static three-mirror cavity shows resonance peaks for exactly these values of the phases. However, as also noted in the case of highfinesse cavities in Section 3, in dynamical cases the peaks appear a few rtt after these resonance points are crossed.

\section{B. Analysis of Results}

We noted in Section 4 that the effective finesse of a threemirror system is the highest when the beam operates in the double-resonance condition (which happens in case aa here) and is the lowest when the beam is on exact antiresonance in the arm cavity but on resonance in the recycling cavity (which happens in case ii). In the following discussion, I refer to these two operating conditions as highest finesse condition and lowest finesse condition, respectively.

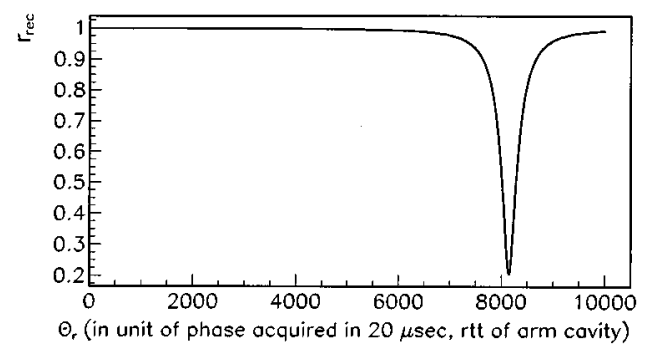

Fig. 25. Equivalent reflectivity of the recycling cavity as a function of the phase offset in the same cavity. The points $(\zeta=) 324$ and 8147 for the phase offset correspond to $\theta_{r}=2 m \pi$ and $\theta_{r}$ $=(2 m+1) \pi$, respectively, as in Figs. 16-23.
The effective finesse as expressed in terms of $r_{\text {rec }}$ can be plotted as a function of $\theta_{r}$ expressed in terms of $\zeta$ (for the same range covered in any of Figs. 16-23), as shown in Fig. 25. As can be seen, the effective finesse is quite high except for a small range around $\zeta=8147$ [where $\theta_{r}$ $=(2 m+1) \pi]$.

On the basis of these understandings, we can explain a few physical effects related to what we observe in Figs. 16-23:

- First peak for the field, D: We observe that as we go from Fig. 16 to Fig. 21 (i.e., from the highest to a lower finesse condition for the peak), the resonance power of the first peak for the field $D$ increases initially (cases aa to $\mathrm{ff}$ ) and then decreases again (cases ff to ii). As we go from case aa to case ff, the effective finesse decreases slowly, the sensitivity to mirror movement decreases, and thus the first peak grows higher. However, for cases gg to kk, the effective finesse is quite low, and although the system is now much less sensitive to the mirror movement, the maximum achievable power (quasi-static) itself can have a low value again for the same reason: having low effective finesse.

- Second peak for the field, B: For case aa (Fig. 16), the $B$ field is on its individual resonance at its second peak. Since the recycling cavity itself has a low finesse, the field evolution follows a typical resonance curve of a low-finesse two-mirror cavity without any oscillation after the resonance point is crossed. It remains so in the next few cases but then starts decreasing again, when its coupling with the $D$ field becomes very important as the point $\theta_{c}=2 n \pi$ comes nearer to $\theta_{r}=(2 m+1) \pi$.

- Second peak for the field, D: For case aa and a few of the subsequent cases, this peak is just the part that leaked into the arm cavity from the individual resonance power of the recycling cavity. This has a typical lowfinesse shape and grows higher as the corresponding peak for the field $B$ decreases from its quasi-static value because of more coupling between two cavities at the operating condition of the second peak.

- First peak for the field, $B$ : In case aa, this peak is just the double-resonance curve, and it experiences the high value of effective finesse of the system with its own oscillations and by its failure to achieve the quasi-static power. It slowly grows in power and sheds its highfinesse appearance as it approaches the $\theta_{r}=(2 m+1) \pi$ point, the individual resonance point of the recycling cavity.

- A Symmetry: As can be expected, the arrangement of the curves is symmetrical about case $\mathrm{hh}$, when the value of $\nabla$ (the value of $\theta_{c}$ when $\theta_{r}=\pi$ ) is $+0.0048 \pi$. That is why case $\mathrm{kk}(\nabla=-0.0204)$ is symmetrical with case $\mathrm{gg}(\nabla=+0.03 \pi)$. From this symmetry one can easily guess what would happen if the point $\theta_{c}=2 n \pi$ is moved between $\theta_{r}=(2 m+1) \pi$ and $\theta_{r}=2 m \pi$.

The only question is why the symmetry is about the case represented by $\nabla=+0.0048$ (case hh) and not about that represented by $\nabla=0.0$ (case ii). This is because of some asymmetry introduced by the dynamical response of the system, i.e., due to the shifting of the resonance curve from the actual resonance point. However, as can be seen from the corresponding quasi-static curve for case ii 
[Fig. 24(b)], the symmetry could have been actually observed to be about case ii, in the case in which the experiments were done in a static three-mirror cavity by stepby-step changes in phase offsets. For similar reasons, this symmetry for the field $B$ is about the case $\mathrm{jj}$, which corresponds to $\nabla=-0.0048$.
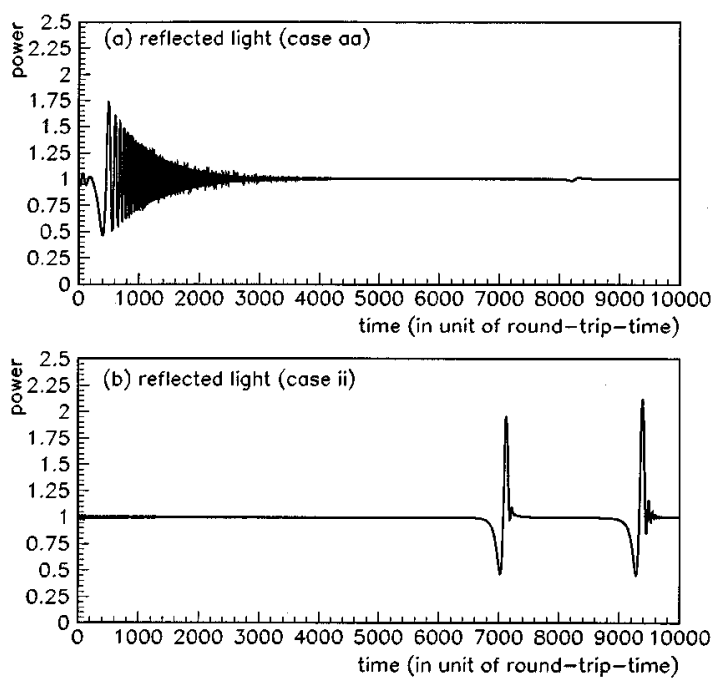

Fig. 26. Reflected light in (a) case aa and (b) case ii.
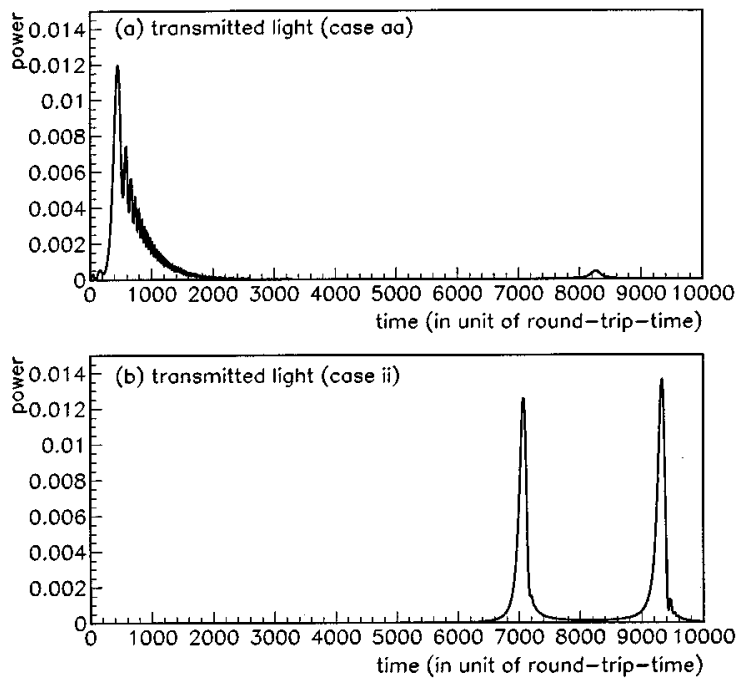

Fig. 27. Transmitted light in (a) case aa and (b) case ii.
- Reflected light: Every resonance point is characterized by many oscillations in the reflected beam from the three-mirror cavity, as shown in Fig. 26. The oscillations are most prominent when the beam is on double resonance (first peak of case aa) and are much less as the operating condition shifts to a state of lower effective finesse (second peak of case aa and peaks of case ii).

- Transmitted light: As can be expected from the analytical expressions, the evolution of peaks of the transmitted beams in the successive figures follows the same kind of pattern as that of the peaks of the field $D$. Figure 27 shows peaks in the transmitted field for two cases, aa and ii. One may note that unlike in the static case, the maximum transmission (among all other peaks) does not occur at the double-resonance condition when the mirrors move.

- Positioning of peaks in quasi-static cases: Let us denote the values of $\theta_{r}$ and $\theta_{c}$ at the first and second peaks, respectively, by

$$
\theta_{r}=2 \pi m+K_{r} \Delta_{\mathrm{i}}^{r}, \quad \theta_{c}=2 \pi n+K_{c} \Delta_{\mathrm{i}}^{c},
$$

where $\mathrm{i}=1,2$ represents the first and second peaks, respectively. The values of these $\Delta$ for both static and dynamical cases are presented in Table 4.

Let us also express the phase of the reflected light from the arm cavity as

$$
\theta_{\text {refl }}=(2 m+1) \pi+K_{r} \Delta_{\mathrm{i}}^{\text {refl }}
$$

and plot $\Delta_{i}^{\text {refl }}$ as a function of phase offset in the arm cavity $\left(\Delta_{\mathrm{i}}^{c}\right)$ in Fig. 28. For reasons to be explained soon, $\Delta_{i}^{\text {refl }}$ is expressed in the same unit as that of $\theta_{r}$, as can be noticed above. It should be noticed that the $(0,0)$ point of this plot corresponds to $\theta_{c}=2 n \pi$ and $\Delta_{\text {refl }}=(2 m$ $+1) \pi$, representing the fact that the phase on reflection from an undercoupled cavity is an odd multiple of $\pi$ on resonance.

By inspecting values of different quantities in Table 4 and referring to Fig. 28, we can arrive at the following conclusion: In the case of a static three-mirror cavity, a peak can always be found whenever $\Delta_{\mathrm{i}}^{c}$ (phase offset in the arm cavity) is such that the corresponding value of $\Delta_{i}^{\text {refl }}$ (phase on reflection from the arm cavity in excess of an odd multiple of $\pi$ ) exactly cancels the value of $\Delta_{\mathrm{i}}^{r}$ (phase offset in recycling cavity in excess of an even multiple of $\pi$ ) at that operating condition, thus making the

Table 4. Phase Offsets in Three-Mirror Coupled Cavity at Positions of the Two Peaks for Various Dynamical and Corresponding Quasi-Static Cases

\begin{tabular}{lrrrrrrrr}
\hline & $\Delta_{1}^{c}(\mathrm{DY})$ & $\Delta_{1}^{r}(\mathrm{DY})$ & $\Delta_{2}^{c}(\mathrm{DY})$ & $\Delta_{2}^{r}(\mathrm{DY})$ & $\Delta_{1}^{c}(\mathrm{QS})$ & $\Delta_{1}^{r}(\mathrm{QS})$ & $\Delta_{2}^{c}(\mathrm{QS})$ & $\Delta_{2}^{r}(\mathrm{QS})$ \\
\hline aa & 126 & 126 & 7938 & -7708 & 0 & 0 & 7936 \\
bb & 68 & 1241 & 6801 & -7672 & -59 & 1114 & 6798 & -7675 \\
cc & -10 & 2493 & 5524 & -7619 & -134 & 2369 & 5520 & -7623 \\
dd & -111 & 3722 & 4273 & -7540 & -230 & 3603 & 4267 & -7546 \\
ee & -265 & 4898 & 3076 & -7407 & -374 & 4789 & 3063 \\
ff & -539 & 5954 & 1998 & -7155 & -629 & 5864 & -7420 \\
gg & -878 & 6546 & 1391 & -6830 & -941 & 6483 & 1973 \\
hh & -1041 & 6718 & 1214 & -6673 & -1095 & 6664 & -7180 \\
ii & -1074 & 6749 & 1183 & -6640 & -1127 & 6696 & 1159 \\
jj & -1109 & 6778 & 1152 & -6607 & -1159 & 6728 & -6878 \\
kk & -1227 & 6868 & 1061 & -6490 & -1271 & 6824 & 1095 & -6728 \\
\hline
\end{tabular}




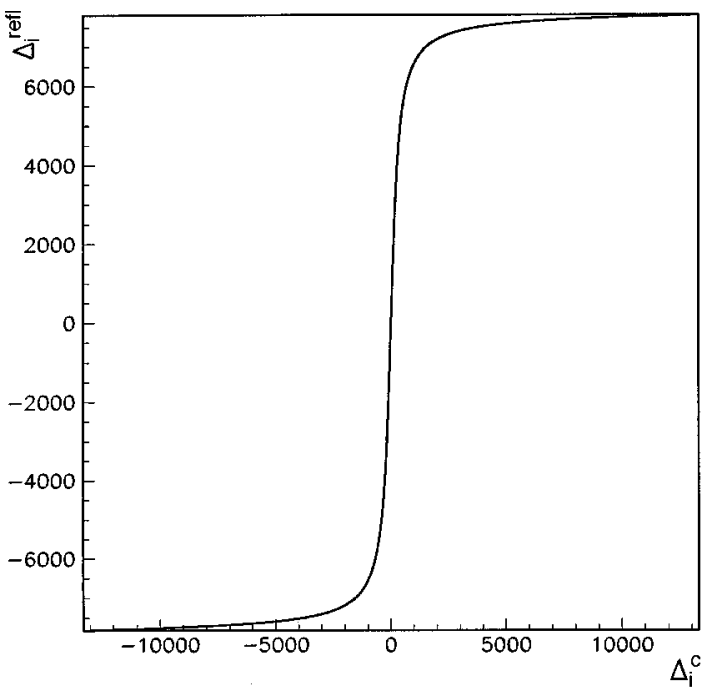

Fig. 28. Phase acquired on reflection [in excess of $(2 m+1) \pi$ ] from the arm cavity as a function of the phase offset (in excess of $2 n \pi)$ in the arm cavity. These are expressed in units of phases acquired by recycling and arm cavities in a round-trip-time of the arm cavity due to mirror motion corresponding to $w_{r}=1.7 \mu \mathrm{m} / \mathrm{s}$ and $w_{c}=1 \mu \mathrm{m} / \mathrm{s}$, respectively.

round-trip phase in the recycling cavity equal to $(2 \mathrm{~m}$ $+1) \pi$, the condition for the individual resonance of the recycling cavity.

The essence of the statement is that a peak always appears whenever the operating condition fulfills the requirement of the individual resonance condition of the recycling cavity, i.e., $\theta_{r}+$ phase on reflection $=(2 m$ $+1) \pi$; the arm cavity is individually resonant only in the double-resonance condition. In all other cases, the phase offset in the arm cavity should change in a suitable way in order to produce a resonance peak.

Notice that a statement like the above was also made at the end of Section 3 of Ref. 11, but their statement bears a restriction equivalent to $K_{c} \Delta_{\mathrm{i}}^{c} \ll 1$ (i.e., phase offset in the arm cavity expressed in radians). The fact that the above rule is always true in the static case without any restriction on phase offset can be tested for each of the cases presented in Table 4.

- Positioning of peaks in dynamical cases: We observe that near double resonance (first peak of case aa) the gap between the dynamical peak $\left[\zeta_{1}(\mathrm{DY})=450\right.$, Table 2] and the quasi-static peak $\left[\zeta_{1}(\mathrm{QS})=324\right.$, Table $3]$ is $\sim 126$. The value of this gap decreases to almost nil for case ii when the operating condition at the point of appearance of the first peak corresponds to a low value of the effective finesse of the system. In conclusion, we can say that even in the dynamical cases, the peaks try to position themselves in accordance with the rule described above; however, when the effective finesse is quite high, the maximum point of the combined power of all partial beams appears at a point somewhat displaced from what would have been their original point of resonance in a static case. This displacement is reduced if either the velocity or the effective finesse decreases.

In this section we noted how combinations of peaks may appear in coupled cavities under general conditions of phase evolution in different constituent cavities. A power-recycled FP-type interferometer is rather a combination of two three-mirror systems that are coupled together by a shared part of their recycling cavities. By observing a small number of details of such successive peaks in these systems during start-up time, one may decide in which way to control the cavities to bring them closer to the desired operating condition before starting the linear/nonlinear locking procedure, thereby saving time and effort. Development of such an algorithm based on this information is currently in progress.

\section{REAL-TIME SIMULATION OF POWER- RECYCLED INTERFEROMETRIC DETECTORS}

In this section I apply all the techniques developed and applied in previous sections to the case of a full-length power-recycled interferometer to develop a fast dynamical simulation code for it. The in-line arm cavity (which receives transmitted light through the beam splitter) and the perpendicular arm cavity (which receives reflected light from the beam splitter) are represented by $X$ and $Y$, respectively; These two letters appear in the subscripts of various quantities for differentiation between the two arm cavities.

We may think of the whole interferometer as a combination of two three-mirror coupled cavity systems, which have a common shared part (that between recycling mirror and beam splitter) in their recycling cavities. The recycling cavity $X$ is the one between the recycling mirror and the input mirror of the in-line arm cavity, whereas the recycling cavity $Y$ is the one between the recycling mirror and the input mirror of the perpendicular arm cavity. Some amount of light always gets exchanged between these two three-mirror systems. In the case of a static interferometer, this exchange rate remains constant; however, in dynamical cases, the rate may vary depending on phase conditions of these systems, as we can see in the equations in the following subsections.

\section{A. Simplified Problem: When Only the End Mirrors Move}

To investigate the nature of the coupling of fields between the two three-mirror systems constituting the whole interferometer, let us first study a simple case with the following assumptions: (i) only the end mirrors move, (ii) the beam splitter is static, (iii) the corresponding mirror reflectivities in both arms are perfectly matched to each other, (iv) the beam splitter is of (exactly) 50:50 type, (v) the round-trip phases in recycling cavities, $\theta_{r y}$ and $\theta_{r x}$, are set to be integral multiples of $2 \pi$, which ensures that whenever a particular arm cavity becomes resonant (i.e., when $\theta_{c y}$ or $\theta_{c x}$ reaches a value of integral multiple of $2 \pi$ ), the corresponding recycling cavity also becomes resonant (by receiving the extra $\pi$ phase from reflection) and the corresponding three-mirror system becomes doubly resonant. The aim of locking the whole interferometer is to make both of the component three-mirror systems doubly resonant for the laser carrier frequency.

These simplifications are made for the time being so that we can concentrate only on the physical effects that are due to dynamical coupling between the two three- 
mirror systems, while avoiding other effects. For such a simple case of a dynamical interferometer, let us write the equations for the intracavity fields with the short-reccav approximation, thereby neglecting lengths $l_{0}, l_{x}$, and $l_{y}$ of parts of the recycling cavities (see Fig. 1), which are too small as compared with the full arm length of the interferometer. The two arm lengths are considered to be same, $L=3000 \mathrm{~m}$. The intracavity fields at the input mirrors and traveling toward the end mirrors inside the respective arm cavities are represented by $D_{y}$ and $D_{x}$. The field at the recycling mirror and traveling toward the beam splitter inside the common shared part of the recycling cavities is represented by $B_{\text {sh }}$. The error analysis of the small-rec-cav approximation in Section 4 gives us the confidence that this should lead to a negligible level of error near resonance for any of the constituent threemirror cavities and thus for the whole interferometer:

$$
\begin{aligned}
B_{\mathrm{sh}}(t)= & t_{1} A+\frac{1}{\sqrt{2}} r_{1} t_{2} r_{3} D_{y}(t-\tau) \exp j\left[\theta_{c y}(t-\tau / 2)\right] \\
& +\frac{1}{\sqrt{2}} r_{1} t_{2} r_{3} D_{x}(t-\tau) \exp j\left[\theta_{c x}(t-\tau / 2)\right] \\
& -r_{1} r_{2} B(t) \\
D_{x}(t)= & \frac{1}{\sqrt{2}} t_{2} B(t)+r_{2} r_{3} D_{x}(t-\tau) \\
& \times \exp j\left[\theta_{c x}(t-\tau / 2)\right] \\
D_{y}(t)= & \frac{1}{\sqrt{2}} t_{2} B(t)+r_{2} r_{3} D_{y}(t-\tau) \\
& \times \exp j\left[\theta_{c y}(t-\tau / 2)\right]
\end{aligned}
$$

Note that the rtt's in both of the arm cavities are assumed to be same and equal to $\tau$. In the actual design of an interferometer, however, the FP cavities in the arms may be physically different in length even by several meters. We can say right away that as long as this difference is less than or of the order of $0.4 \%$ (the length of recycling cavity as compared with that of the arm in the case of VIRGO), the error would remain at the same negligible level as that of small-rec-cav approximation.

These equations can be rearranged and written as

$$
\begin{aligned}
B_{\mathrm{sh}}(t)= & \frac{r_{1} t_{2} r_{3}}{\sqrt{2}\left(1+r_{1} r_{2}\right)} D_{y}(t-\tau) \exp j\left[\theta_{c y}(t-\tau / 2)\right] \\
+ & \frac{r_{1} t_{2} r_{3}}{\sqrt{2}\left(1+r_{1} r_{2}\right)} D_{x}(t-\tau) \exp j\left[\theta_{c x}(t-\tau / 2)\right] \\
+ & \frac{t_{1} A}{\left(1+r_{1} r_{2}\right)}, \\
D_{y}(t)= & \mathscr{b}+r_{3} \beta D_{y}(t-\tau) \exp j\left[\theta_{c y}(t-\tau / 2)\right] \\
& +r_{3} \mathscr{C D} D_{x}(t-\tau) \exp j\left[\theta_{c x}(t-\tau / 2)\right], \\
D_{x}(t)= & \not b+r_{3} \beta D_{x}(t-\tau) \exp j\left[\theta_{c x}(t-\tau / 2)\right] \\
& +r_{3} \& D_{y}(t-\tau) \exp j\left[\theta_{c y}(t-\tau / 2)\right],
\end{aligned}
$$

where

$$
\begin{aligned}
& \mathscr{C}=\frac{t_{1} t_{2} A}{\sqrt{2}\left(1+r_{1} r_{2}\right)}, \\
& \beta=r_{2}+\mathscr{C}=r_{2}+\frac{r_{1} t_{2}{ }^{2}}{2\left(1+r_{1} r_{2}\right)}, \\
& \mathscr{C}=\frac{r_{1} t_{2}{ }^{2}}{2\left(1+r_{1} r_{2}\right)} .
\end{aligned}
$$

The numerical values of $\beta$ and $\mathscr{C}$ for the chosen parameter values for VIRGO are $\approx 0.969367$ and $\approx 0.029367$, respectively. In the case in which both arm cavities change in length in the same manner, their fields also evolve in the same way, and we can set $D_{y}=D_{x}$ and regain the three-mirror coupled cavity equation. We can then identify

$$
r_{\text {rec }}=\beta+\mathscr{C} \approx 0.998733 .
$$

Under such a condition, the nature of the field evolution shows a high-finesse behavior corresponding to $r_{c}$ $=r_{\text {rec }}$, as shown in Fig. 29(a) for either $D_{x}$ or $D_{y}$. One may note that the field evolution behavior is exactly like that in Fig. 6 for the field $F$ in a two-mirror cavity of $r_{c}$ $=0.998733$ or the field $D$ in the three-mirror cavity considered in Section 4, the only difference being in power level-a factor of one half-originating from the presence of the beam splitter in the interferometer.

If the motion in the two arm cavities is out of phase, each arm cavity becomes resonant on its own at different points in time and this resonance shows a low-finesse behavior corresponding to $r_{c}=\beta$, as shown in Fig. 29(b). The contribution of the coupling of the nonresonant cavity to the resonant one is negligible in that case. Whenever the values of $\theta_{c y}$ and $\theta_{c x}$ become close together and move together toward the resonance point, fields in the arm
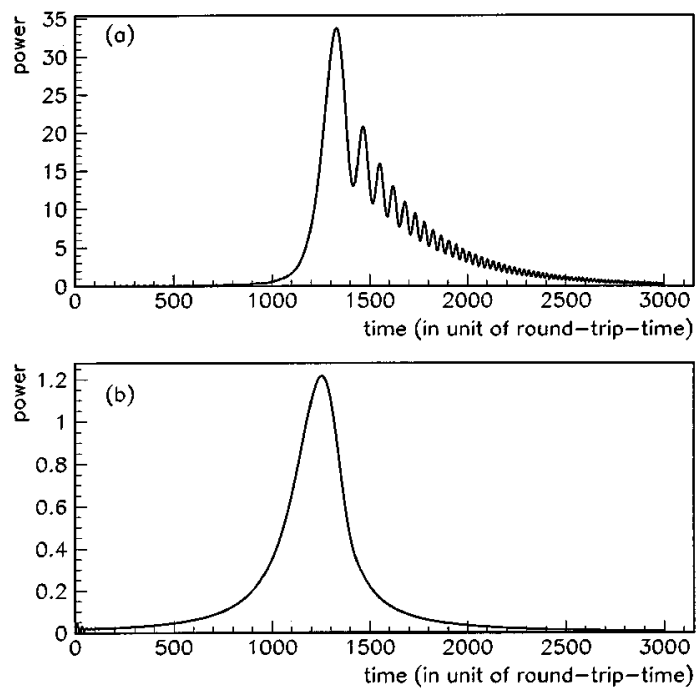

Fig. 29. (a) Resonance peak for either of the fields $D_{y}$ or $D_{x}$ when the three-mirror systems become doubly resonant simultaneously. (b) Peak in power of either $D_{y}$ or $D_{x}$ when only the corresponding three-mirror system becomes doubly resonant but the other three-mirror system remains far out of resonance. In both figures the input power is one unit. 
cavities start becoming coupled to each other and the term with the coupling factor $\mathscr{C}$ becomes important.

We may thus conclude that between the two extreme cases described above, whenever the two sets of threemirror coupled cavities cross their individual resonance points (double or other types) very closely in time, the fields strongly couple with each other and their evolution always shows characteristics typical of two-mirror cavities with a value of $r_{c}$ between $\beta$ and $\beta+\mathscr{C}$. The exact profiles of the resonance peaks in individual arm cavities may, however, differ from each other depending on how closely in time and with what velocities of their constituent mirrors the two systems cross their resonance points.

An increase in the finesse of the arm cavities leads to an increased value of $\beta$ but a reduced value for $\mathscr{C}$ (one should note, however, that if arm cavity finesse is increased, one also has to change $r_{1}$ to have optimum recycling of light). But that does not make coupling between the arms on resonance less important than in the case of low-finesse arm cavities, because the power stored is much higher in high-finesse arm cavities.

The situation in which the fields in the arms couple with each other is the one that is important for the purpose of locking. To simulate such or other situations, the following algorithm is developed.

\section{B. Simulation Procedure for the Whole Interferometer}

The simulation procedure to be described here is based on the state-vector representation of the intracavity fields of the full interferometer. This particular method of solving problems has been used in many multi-input/multioutput cases in the field of digital filtering and signal processing (see Chaps. 4 and 12 of Jackson ${ }^{7}$ ). The method is presented in three parts: first, the equations for the simple case of the last subsection is written in statevector representation. Next, the equations are generalized by incorporating dynamical changes in the recycling cavity and also other asymmetries that are due to differing mirror reflectivities. Finally, the simulation procedure for both of these cases are discussed.

\section{Equations for the Simple Case}

In order to express the main points of the simulation procedure in an easier way, let us first consider the simple case discussed in Subsection 6.A and write Eqs. (59) and (60) with the following state-vector representation:

$$
\mathbf{D}(t+\tau)=\mathbf{A}+\mathbf{R} \mathscr{E}(t+\tau / 2) \mathbf{D}(t),
$$

where

$$
\begin{aligned}
\mathbf{D}(t) & =\left[\begin{array}{l}
D_{y}(t) \\
D_{x}(t)
\end{array}\right], \\
\mathbf{A} & =\mathscr{C}\left[\begin{array}{l}
1 \\
1
\end{array}\right], \\
\mathbf{R} & =r_{3}\left[\begin{array}{ll}
\beta & \mathscr{C} \\
\mathscr{C} & \beta
\end{array}\right]\left[\begin{array}{cc}
\exp j\left(\phi_{c y}\right) & 0 \\
0 & \exp j\left(\phi_{c x}\right)
\end{array}\right],
\end{aligned}
$$

$$
\mathscr{E}(t)=\left[\begin{array}{cc}
\exp j\left(\frac{4 \pi}{\lambda} v_{3 y} t\right) & 0 \\
0 & \exp j\left(\frac{4 \pi}{\lambda} v_{3 x} t\right)
\end{array}\right],
$$

where it is assumed that for a short time the end mirrors are moving with velocities $v_{3 y}$ and $v_{3 x}$, respectively; $\phi_{c y}$ and $\phi_{c x}$ are initial round-trip-phase offsets in arm cavities.

\section{Equations for the Generalized Case}

When all mirrors move, we can write the coupled equations of the intracavity fields in the arms by also incorporating all asymmetries that are due to differing mirror reflectivities and the moving beam splitter as

$$
\mathbf{D}(t+\tau)=\mathbf{A}(t+\tau)+\mathbf{R}(t+\tau) \mathscr{E}(t+\tau / 2) \mathbf{D}(t) .
$$

Various quantities in Eq. (70) are defined as follows. Note that, in the subscripts of these quantities, letters $c$ and $r$ represent arm cavity and recycling cavity, respectively; subscripts $1,2,3$, and $b$ represent recycling mirror, input mirror, end mirror, and beam splitter, respectively; as already stated, $x$ and $y$ refer to various quantities for the three-mirror systems $X$ and $Y$, respectively:

$$
\begin{aligned}
& \mathbf{A}(t)=\frac{t_{1} A}{\chi(t)}\left[\begin{array}{c}
r_{b} t_{2 y} \exp j\left[\Theta_{r y \uparrow}(t)\right] \\
t_{b} t_{2 x} \exp j\left[\Theta_{r x}(t)\right]
\end{array}\right], \\
& \chi(t)=1+r_{1} r_{b}^{2} r_{2 y} \exp j\left[\Theta_{r y \uparrow}(t)+\Theta_{r y \downarrow}(t)\right] \\
& +r_{1} t_{b}^{2} r_{2 x} \exp j\left[2 \Theta_{r x}(t)\right], \\
& \mathbf{R}(t)=\left[\begin{array}{cc}
\beta_{y} & \mathscr{C}_{x} \\
\mathscr{C}_{y} & \beta_{x}
\end{array}\right]\left[\begin{array}{cc}
\exp j\left(\phi_{c y}\right) & 0 \\
0 & \exp j\left(\phi_{c x}\right)
\end{array}\right], \\
& \mathscr{E}(t)=\left[\begin{array}{cc}
\exp j\left(\frac{4 \pi}{\lambda} W_{c y} t\right) & 0 \\
0 & \exp j\left(\frac{4 \pi}{\lambda} W_{c x} t\right)
\end{array}\right], \\
& \beta_{y}=r_{2 y} r_{3 y}+\frac{1}{\chi(t)} r_{1} r_{b}^{2} t_{2 y}^{2} r_{3 y} \\
& \times \exp j\left[\Theta_{r y \uparrow}(t)+\Theta_{r y \downarrow}(t)\right] \text {, } \\
& \beta_{x}=r_{2 x} r_{3 x}+\frac{1}{\chi(t)} r_{1} t_{b}^{2} t_{2 x}^{2} r_{3 x} \exp j\left[2 \Theta_{r x}(t)\right], \\
& \mathscr{C}_{x}=\frac{1}{\chi(t)} r_{1} r_{b} t_{b} t_{2 y} t_{2 x} r_{3 x} \exp j\left[\Theta_{r x}(t)+\Theta_{r y \uparrow}(t)\right], \\
& \mathscr{C}_{y}=\frac{1}{\chi(t)} r_{1} r_{b} t_{b} t_{2 y} t_{2 x} r_{3 y} \exp j\left[\Theta_{r y \downarrow}(t)+\Theta_{r x}(t)\right] .
\end{aligned}
$$

The phase factors in the above equations can be expressed in the following form if we assume that for a short span of time $N \tau$ (where $N$ is some number to be determined numerically), all mirrors are moving with constant velocities [note: this assumption on arm cavities has already been used in writing Eq. (70)]: 


$$
\begin{aligned}
\Theta_{r y \uparrow}(t) & =\phi_{r y} / 2+\frac{2 \pi}{\lambda}\left(W_{r y} t+V_{b x} t\right), \\
\Theta_{r y \downarrow}(t) & =\phi_{r y} / 2+\frac{2 \pi}{\lambda}\left(W_{r y} t+V_{b y} t\right), \\
\Theta_{r x}(t) & =\phi_{r x} / 2+\frac{2 \pi}{\lambda} W_{r x} t, \\
\theta_{c y}(t) & =\phi_{c y}+\frac{4 \pi}{\lambda} W_{c y} t, \\
\theta_{c x}(t) & =\phi_{c x}+\frac{4 \pi}{\lambda} W_{c x} t,
\end{aligned}
$$

where $\phi$ 's are initial round-trip phase offsets in various cavities and $W$ 's are relative velocities defined as

$$
\begin{aligned}
& W_{r y}=V_{2 y}-V_{1}, \\
& W_{r x}=V_{2 x}-V_{1}, \\
& W_{c y}=V_{3 y}-V_{2 y}, \\
& W_{c x}=V_{3 x}-V_{2 x} .
\end{aligned}
$$

All the $V$ 's above are magnitudes of velocities of the corresponding mirrors with respect to the initial position of the beam splitter; $V_{b x}, V_{b y}$ are components of the velocity of the beam splitter with respect to its initial position, as shown Fig. 30. It should be noted that if the beam splitter moves, light beams do not retrace their paths while returning to the beam splitter. This might lead to distortion of beams in the transverse direction and thus generation of higher-order modes. In this paper, however, we confine our attention to the longitudinal effects.

\section{Simulation Steps}

Step 1. Time is sliced into equal intervals of width $\Delta$ $=N \tau$, so that any time $t_{i}=i N \tau$, where $i$ is an integer.

Step 2. During the time interval $\left(t_{i}, t_{i+1}\right)$, the rates of the changes of phase offsets in arm cavities, $v_{3 x}$ and $v_{3 y}$ (simple case) or $W_{r y}, W_{r x}, W_{c y}, W_{c x}, V_{b y}$, and $V_{b x}$ (generalized case), are assumed to be constant.

Step 3. The phases $\phi_{c x}, \phi_{c y}$ are fixed to the following constant values during $\left(t_{i}, t_{i+1}\right)$ :

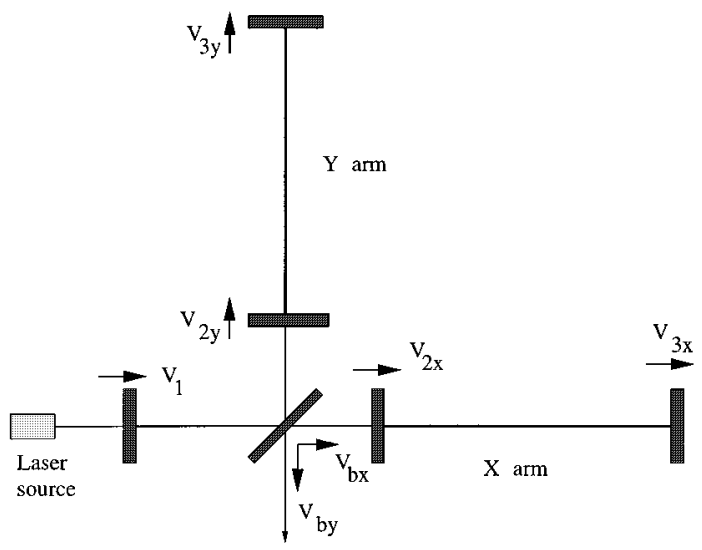

Fig. 30. Notation for the mirror velocities with respect to the initial position of the beam splitter. The arrows indicate positive signs for V's in Eqs. (84)-(87).

$$
\begin{aligned}
& \phi_{c x}=\theta_{c x}\left(t_{i}\right), \\
& \phi_{c y}=\theta_{c y}\left(t_{i}\right) .
\end{aligned}
$$

Step 4. (FTF for the generalized case). The matrices $\mathbf{R}$ and $\mathbf{A}$ are set to the following constant matrices during the same time interval:

$$
\begin{aligned}
& \mathbf{R}=\frac{1}{2}\left[\mathbf{R}\left(t_{i}\right)+\mathbf{R}\left(t_{i+1}\right)\right], \\
& \mathbf{A}=\mathbf{A}\left(t_{i+1}\right) .
\end{aligned}
$$

Step 5. (JAS). Now by performing the equivalent algebraic operations of JAS as described in Section 3 on either Eq. (65) (simple case) or Eq. (70) (for which $\mathbf{R}$ and $\mathbf{A}$ are now constant matrices), we can arrive at the following equation equivalent to Eq. (27):

$$
\begin{aligned}
\mathbf{D}(t+N \tau)= & \mathbf{R}^{N} \mathbf{E D}(t)+(\mathbf{U}-\mathbf{R})^{-1}\left[\left(\mathbf{U}-\mathbf{R}^{N}\right)\right. \\
& \left.+\mathbf{R S} \boldsymbol{\xi}-\frac{1}{2}\left(N^{2}-1\right) \mathbf{R}^{N} \boldsymbol{\xi}\right] \mathbf{A},
\end{aligned}
$$

where

$$
\begin{aligned}
\mathbf{U}= & {\left[\begin{array}{ll}
1 & 0 \\
0 & 1
\end{array}\right], } \\
\mathbf{E}= & {\left[\begin{array}{cc}
\exp \left(\xi_{y} N^{2} / 2\right) & 0 \\
0 & \exp \left(\xi_{x} N^{2} / 2\right)
\end{array}\right], } \\
\boldsymbol{\xi}= & {\left[\begin{array}{cc}
\xi_{y} & 0 \\
0 & \xi_{x}
\end{array}\right], } \\
\mathbf{S}= & (\mathbf{U}-\mathbf{R})^{-1}\left[\left(N-\frac{1}{2}\right) \mathbf{U}-1.5 \mathbf{R}^{N-1}\right] \\
& +(\mathbf{U}-\mathbf{R})^{-2}\left(\mathbf{R}^{N-1}-\mathbf{R}\right), \\
\xi_{y}= & j \frac{4 \pi W_{c y} \tau}{\lambda} \\
\xi_{x}= & j \frac{4 \pi W_{c x} \tau}{\lambda} .
\end{aligned}
$$

Equation (92) is now applied to calculate the value of the field inside the arm cavities.

Step 6. In the next step, the same procedure is performed by replacing $i \leftarrow i+1$ and with changed (if these change) mirror velocities.

The error levels are found to be almost at the same levels as in three-mirror cavities (Subsection 4C) when the two cavities achieve either the highest (Fig. 14) or the lowest (Fig. 15) finesse conditions at the same time.

Figure 31(a) shows the dynamical curve for the field $D_{y}$ for the case in which the three-mirror system $Y$ becomes doubly resonant ahead of system $X$ with a time gap corresponding to a phase difference of $\pi / 100$, where $W_{r y}$ $=W_{c y}=W_{r x}=W_{c x}=1 \mu \mathrm{m} / \mathrm{s}$ and the beam splitter is assumed to be static. The relative error by DFA compared with equations based on only the small-rec-cav approximation is shown in Fig. 31(b). The difference between power levels of $D_{y}$ and $D_{x}$ is shown in Fig. 32 .

Figure 33(a) shows the peak in field $D_{y}$ when the threemirror system $Y$ gets to the lowest finesse condition of "antiresonant in the arm cavity but resonant in the recycling cavity" ahead of system $X$ with a time gap corresponding to a phase difference of $\pi / 100$, where $W_{r y}$ $=W_{c y}=W_{r x}=W_{c x}=1 \mu \mathrm{m} / \mathrm{s}$ and the beam splitter is 
assumed to be static. The relative error by the DFA compared with equations based on only the small-rec-cav approximation is shown in Fig. 33(b). Note that in this case no difference between power levels of $D_{x}$ and $D_{y}$ could be found even at a level of $10^{-4}$ units of power. This is another manifestation of the low-finesse characteristics of the operating condition for the beam. Under this condition partial beams do not get stored for a long time and so do not cause any difference between fields in arm cavities.

\section{CONCLUDING REMARKS}

It may be noted that the discussion so far has been confined to studying the response of the systems to a beam of a single frequency. However, for the purpose of controlling the mirrors, one needs to employ some sensing scheme based on phase modulation of light. Under these schemes ${ }^{19}$ the phase of the laser light is modulated at a radio frequency. The effect of this modulation is to impose two sidebands on the laser, $\omega_{0} \pm \omega_{m} ; \omega_{0}, \omega_{m}$ are the carrier frequency and the modulation frequency, respectively. The lengths, $l_{0}, l_{y}$, and $l_{x}$ (in Fig. 1), are so chosen that both the carrier and the sidebands are resonant in the recycling cavity. The operating condition of the sidebands in the arm cavity is generally chosen to be somewhere out of resonance; the best condition seems to be the exact antiresonance. The difference between $l_{y}$ and $l_{x}$ should be suitably chosen so that it allows sideband light to be transmitted to the output port while that port remains dark for the carrier. If, for some reason, the arm lengths of the interferometer become distorted, some amount of carrier light exits the outport port, where it beats against the sidebands. This light is detected and demodulated with a mixer. The signal thus generated is called the Pound-Drever signal, ${ }^{20}$ which is then used to feed back to the laser frequency and/or the mirror control system to bring the system back to the perfect operating condition. This signal is linear with respect to either frequency or length deviation in a small range around the perfect-resonance condition. This range is wider for a system of lower effective finesse, and thus controlling is relatively easier in such a case.

After this brief introduction to the locking schemes, I now summarize some important conclusions of this paper.

\section{A. Physical Effects}

(a) A power-recycled interferometer with FP arm cavities can be thought of as a combination of two three-mirror systems that are coupled to each other through a shared part of their recycling cavities. Field evolution in the arm cavity of any of these dynamical three-mirror systems can be shown to be equivalent to that in a twomirror cavity with changing finesse. An interesting analogy has thus been established between the evolution of the field in a dynamical two-mirror cavity and the evolution of the arm cavity fields in a full interferometer when the latter are given an analytical representation with state vectors.

(b) We may give a quantitative representation of the response of a three-mirror system by the finesse or, equivalently, by the amplitude reflectivity of the input mirror $r_{c}$ of an equivalent two-mirror cavity whose end mirror has a fixed value of high reflectivity. In an interferometer the $r_{c}$ of any of the two coupled three-mirror systems can be divided into two parts: $r_{c} \approx \beta+\mathscr{C}$ in a long range around the desired operating condition of double resonance. The quantities $\beta$ and $\mathscr{C}$ depend on various parameters of the mirrors and depend on time only through the evolution of the phase offsets in the corresponding recycling cavity. The coupling factor $\mathscr{C}$ is always very small but is important in terms of making that small contribution in $r_{c}$ that might change the response of a threemirror system from a lower-finesse to a higher-finesse one.

(c) In the case in which the two three-mirror systems are achieving double resonance at well-separated points in time, the evolution of fields in each system is similar to that of an equivalent two-mirror cavity with $r_{c}=\beta$ and thus shows a lower-finesse behavior. However, if both of the systems achieve double resonance at the same time (which is the desired operating condition for the carrier), the evolution shows a high-finesse behavior corresponding to $r_{c}=\beta+\mathscr{C}$ in both cavities.

(d) A three-mirror system may show peaks under various dynamical conditions of its two constituent cavities. These peaks were studied in Section 5 and a rule was established. It was shown that such peaks appear whenever the recycling cavity becomes resonant; the doubleresonance condition is just one of the various possibilities that may ensure this. In a power-recycled interferometer, the evolution of fields resulting from coupling between a double-resonance peak and a wrong peak or between two wrong peaks from the two three-mirror systems shows a behavior corresponding to $r_{c}$ in between $\beta<r_{c}<\beta+\mathscr{C}$.

(e) Start-up problem. The locking schemes based on phase modulation of light work only when the whole interferometer is very close to its desired resonant condition. Thus one problem with the power-recycled interferometer with suspended mirrors is to bring all of its constituent cavities close to the operating condition starting from time zero, so that the Pound-Drever signal is generated. We may note that if the mirrors move slowly, then most of the time under the start-up condition the field evolution in arm cavities approximately obeys Eqs. (59) and (60) written for an interferometer with $\theta_{r x}$ and $\theta_{r y}$ set to $2 m \pi$. The nature of field evolution deviates considerably from these two equations when the beam is close to the individual resonance point [i.e., approximately $\pm 0.2 \pi$ around $\theta_{r x}$ and/or $\left.\theta_{r y}=(2 m+1) \pi\right]$ of the recycling cavity, whereas the finesse changes quite rapidly. So when mirrors move slowly, we can expect that at least for a small range of time near the desired operating condition we can use Eqs. (59) and (60) to describe the evolution of fields without making much error. It might thus be advantageous if we divide the start-up procedure in two parts. ${ }^{21}$ In step 1 we may try to lock only the three-mirror cavity $Y$ while keeping the $X$ arm cavity out of resonance. While locked, the $Y$ arm cavity will have a constant power corresponding to a two-mirror cavity with input mirror amplireflectivity, $r_{c}=\beta \approx 0.96937 \ldots$ for VIRGO. From Eqs. (59) and (60) we can see that trying to lock the whole interferometer together is like trying to 
lock a high-finesse two-mirror cavity with $r_{c}=\beta+\mathscr{C}$ $=0.998733 \ldots$ (for VIRGO), while keeping control over four cavities coupled together. Now step 1 is like locking a two-mirror cav with $r_{c}=\beta=0.96937 \ldots$ (a lowerfinesse one), while keeping control over two cavities coupled together. The three-mirror system $Y$ is chosen to be locked in step 1 to take care of the extra 2 degrees of freedom of beam-splitter movement right at the beginning so that we can avoid them in the relatively tougher step 2 .

Now, in step 2 when we try to lock the three-mirror cavity $X$, the task will be equivalent to locking a twomirror cavity with $r_{c}=0.998733 \ldots$ but keeping control of only two mirrors of the in-line arm cavity. We can take advantage of the reduced number of degrees of freedom and increased linear region in the Pound-Drever signal in step 1 as a result of the reduced finesse of the system. In step 2 the linear region is reduced to what the full interferometer originally had, but the task becomes relatively easier because most of the degrees of freedom have already been taken care of in step 1. A complete end-toend simulation including the servo control system needs to be developed to test the merits and demerits of the above proposal.
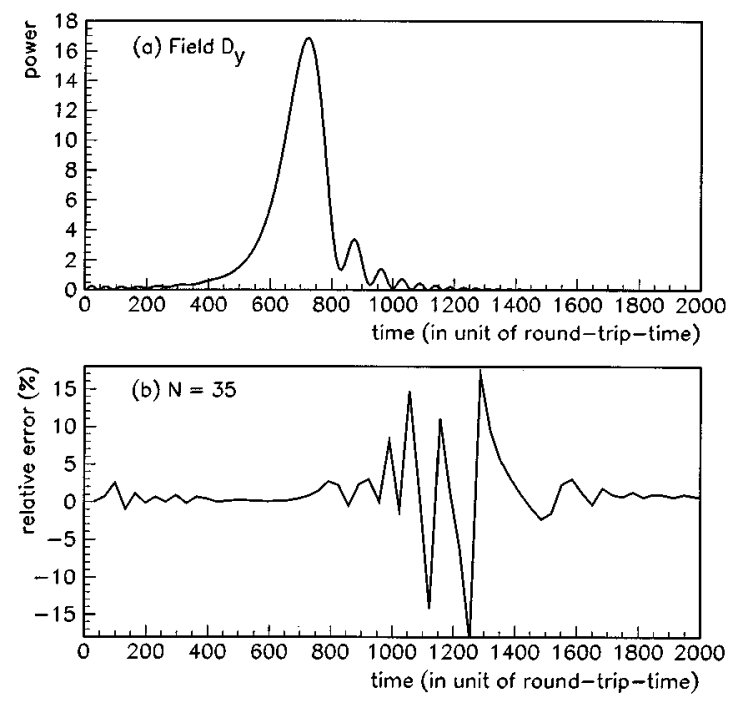

Fig. 31. (a) Resonance peak for the field $D_{y}$ when three-mirror system $Y$ becomes doubly resonant ahead of system $X$, with a time gap equivalent to a phase difference of $\pi / 100$, while the mirrors move such that $W_{r y}=W_{r x}=W_{c x}=W_{c y}=1 \mu \mathrm{m} / \mathrm{s}$ and the beam splitter is assumed to be static. Input power is one unit. (b) Relative error by the DFA based on JAS and FTF with respect to only the small-rec-cav approximation in computing such a peak. The relative error increases for a short time while the field amplitude is very small; this increase is thus unimportant.

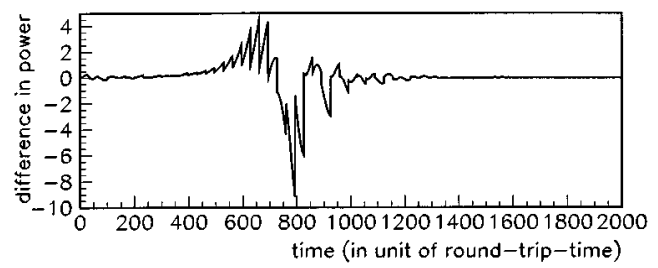

Fig. 32. Difference between resonant power levels of fields $D_{y}$ and $D_{x}$ under the condition described for Fig. 31 .
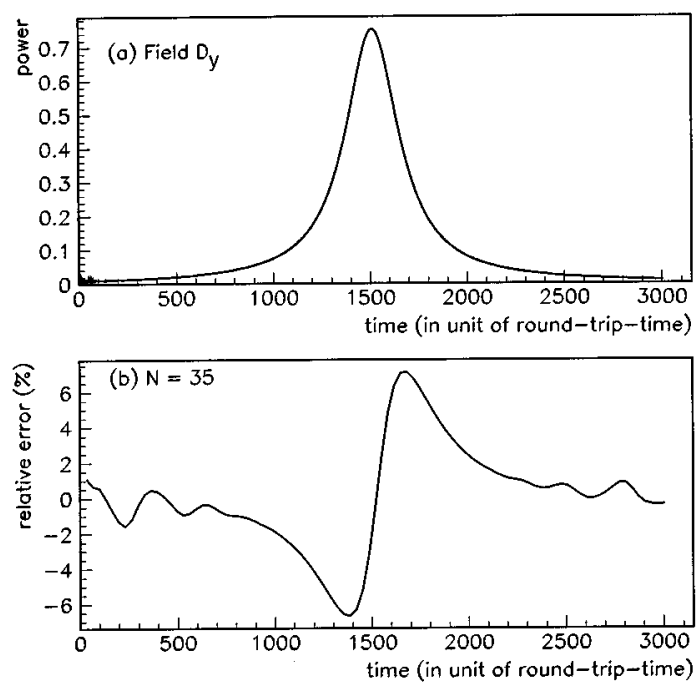

Fig. 33. (a) Peak in power of field $D_{y}$ when three-mirror system $Y$ gets to the condition of "resonance in recycling cavity but exact antiresonance in arm cavity" ahead of system $X$, with a time gap equivalent to a phase difference of $\pi / 100$, while mirrors move such that $W_{r y}=W_{r x}=W_{c x}=W_{c y}=1 \mu \mathrm{m} / \mathrm{s}$ and the beam splitter is assumed to be static. Input power is one unit. (b) Relative error by the DFA based on JAS and FTF with respect to only the small-rec-cav approximation in computing such a peak.

\section{B. Computational Speed}

The simulation program developed here with a digital filtering approach is quite fast and is applicable with tolerable error level to a full-length interferometer with its cavities under general dynamical conditions.

(a) We may write a code for the whole interferometer with the following considerations: (i) It should work under the general operating condition of the interferometer and not just for the case in which it is very near the locking condition; (ii) the operating condition for sidebands can be chosen to be anywhere; (iii) rates of changes in cavity lengths are of the order of $1 \mu \mathrm{m} / \mathrm{s}$.

Such a code written in $C$ language using the DFA based on JAS and FTF with $N=50$ runs faster than real time in a DEC ALPHA workstation; the computational time is approximately $80 \%$ of real time for calculating the dynamical evolution of fields at various locations of the VIRGO interferometer.

(b) The code can be made approximately two times faster than this if (i) the operating condition of the sidebands is chosen to be the lowest-finesse condition (i.e., resonant in recycling cavities but antiresonant in arm cavities) or some other point well inside $\pm 0.2 \pi$ around this (most probably this will be the choice for all longbaseline detectors; in Sections 4 and 6, investigations were made for a beam near such an operating condition) and (ii) we are interested in calculating dynamical-field evolution only near the desired operating condition in which the Pound-Drever signal can be generated.

Since near such a condition the field evolution shows a low-finesse behavior, we can write the part of the code for the sidebands by using the computationally quite inexpensive DFA with the perturbative approach (Section 2) rather than the DFA with JAS. We just need to apply the equivalent matrix equations corresponding to Eqs. 
(13) and (18) instead of Eq. (94) in step 5 in Subsection 6.B.3. Although the error consideration remains the same, two thirds of the code can be made approximately three times more efficient in such a way that the full program runs at a speed approximately two times faster than the general code described in item (a).

(c) When locking is achieved, if we are sure that the system will not go very far from the operating condition, we may switch over to a program somewhat faster than the codes described above by getting rid of the step of freezing-the-finesse in the part of the code for the carrier, since we know that near double resonance the finesse of the system for the carrier changes very little and very slowly.

(d) If the speeds of the mirror can be slowed down considerably, we can go somewhat faster by increasing the length of our jump in time, i.e., increasing the value of $N$ to a certain extent.

(e) These codes have been studied specifically for the VIRGO interferometer, whose arm cavities are of low finesse $(\approx 50)$. There will be very little change in the error consideration and thus in the computational speed of the codes if cavities with higher finesse are incorporated into the arms. Numerical investigation shows that the DFA based on JAS works quite well when applied to interferometers with arm cavities of much higher finesse.

\section{Further Research}

It should be noted that none of the codes addresses the transverse effects on the laser beam. Research on incorporating higher-order effects is currently in progress. In addition, research on testing the computational speed and error of another method of simulating the full interferometer based on the DFA with JAS but summing up the most significant partial beams ${ }^{22}$ coupled between two arm cavities (instead of using the algebra of state-vector representation) is currently in progress and will be communicated in the future.

It needs to be seen how this method works for other potential optical configurations for interferometric gravitational wave detectors: dual recycled, ${ }^{23}$ resonant sideband detecting, ${ }^{12}$ etc. However, an interesting problem is trying this method in Sagnac interferometers ${ }^{24}$ that incorporate dynamical ring cavities in both arms, since the nature of coupling between fields in two arms of such an interferometer is completely different from that in a Michelson topology. The investigation shows that the digital filtering technique with some modifications in its mathematical structure works quite well in Sagnac topology also. The results will be communicated elsewhere.

The most important task ahead is to incorporate these codes inside a complete end-to-end simulation program for the whole interferometer to test the start-up procedure and servo control techniques in the spirit of what has been done by the LIGO team ${ }^{3}$ for a two-mirror cavity.

\section{ACKNOWLEDGMENTS}

I am especially thankful to P. Hello and J.-Y. Vinet for the initial motivation they provided me and for many useful discussions and critical comments. It is also my pleasure to thank M. Barsuglia, F. Bondu, A. Brillet, F. Cavalier, R. Chiche, H. Heitmann, F. Lediberder, C. N. Man, M. Pham Tu, and E. Tournie for comments and clarifications. This work is supported by Centre International des Etudiants et Stagiaires, France.

Present address: TAMA Project, National Astronomical Observatory, Mitaka 181, Japan. Address after November 30, 1997: LIGO Project, Caltech, Pasadena, California 91125 .

\section{REFERENCES}

1. Papers in Proceedings of the International Conference on Gravitational Waves: Sources and Detectors, I. Ciufolini and F. Fidecaro, eds. (World Scientific, Singapore, 1997).

2. R. W. P. Drever, "Interferometer detectors for gravitational radiation," in Gravitational Radiation, N. Deruelle and T. Piran, eds. (North-Holland, Amsterdam, 1983).

3. J. Camp, L. Sievers, R. Bork, and J. Heefner, "Guided lock acquisition in a suspended Fabry-Perot cavity," Opt. Lett. 20, 2463-2465 (1995).

4. VIRGO collaboration, VIRGO: Final Design (Istituto Nazionale di Fisica Nucleare, Pisa, Italy, 1995).

5. B. Bhawal, "Global Control Document: dynamical simulation of cavity using a digital filtering approach," VIRGO Rep. VIR-NTS96-016 (Laboratoire de l'Accélérateur Linéaire, Orsay, France, 1996).

6. S. Song, "Recycling and squeezing in high-precision optical measurements," Ph.D. dissertation (University of Southern California, Los Angeles, Calif., 1994).

7. A. Bateman and W. Yates, Digital Signal Processing Design (Pitman, London, 1988); L. B. Jackson, Digital Filters and Signal Processing (Kluwer Academic, Boston, 1996).

8. F. Cavalier, P. Hello, and J.-Y. Vinet, "Global Control Document: dynamical simulation of cavities," VIRGO Rep. VIR-NTS95-037 (Laboratoire de l'Accélérateur Linéaire, Orsay, France, 1995).

9. B. Bhawal, "Global Control Document: dynamical simulation of 3-mirror coupled cavity using a digital filtering approach," VIRGO Rep. VIR-NTS96-033 (Laboratoire de l'Accélérateur Linéaire, Orsay, France, 1996).

10. A. E. Seigmann, Lasers (University Science, Mill Valley, Calif., 1986), pp. 524-530.

11. P. Fritschel, D. Shoemaker, and R. Weiss, "Demonstration of light recycling in a Michelson interferometer with Fabry-Perot cavities," Appl. Opt. 31, 1412-1418 (1992).

12. J. Mizuno, "Comparison of optical configurations for laserinterferometric gravitational-wave detectors," Ph.D. dissertation (Max-Planck Institut für Quantenoptik, Garching, Germany, 1995), Chap. 4.

13. P. K. Fritschel, "Techniques for laser interferometric gravitational wave detectors," Ph.D. dissertation (Massachusetts Institute of Technology, Cambridge, Mass., 1992), Secs. 5.2, 5.3 .

14. M. W. Regehr, "Signal extraction and control for an interferometric gravitational wave detector," Ph.D. dissertation (California Institute of Technology, Pasadena, Calif., 1995), Sec. 5.4

15. D. Redding, "Mathematical description of the LIGO singlemode acquisition code," LIGO internal Rep. T-960171-00-D (LIGO project, California Institute of Technology, Pasadena, Calif., 1996).

16. F. Marion, in "Minutes of the electronics and software meeting at Annecy, France," B. Mours, ed., VIRGO Rep. VIR-NTS96-037 (Laboratoire d'Annecy de Physique des Particules, Annecy, France, 1996).

17. B. Bhawal, "Global Control Document: freezing-thefinesse-a technique used for fast simulation of 3-mirror coupled cavity," VIRGO Rep. VIR-NOT-LAL-1390-049 (Laboratoire de l'Accélérator Linéaire, Orsay, France, 1996).

18. B. Bhawal, "What happens when all mirrors move in a 3- 
mirror coupled cavity," VIRGO Rep. VIR-NOT-LAL-1390057 (Laboratoire de l'Accélérator Linéaire, Orsay, France, 1996).

19. R. Weiss, "Electromagnetically coupled broadband gravitational antenna," Q. Prog. Rep. Res. Lab. Electron. MIT 105, 54-76 (1972); C. N. Man, D. Shoemaker, M. Pham Tu, and D. Dewey, "External modulation technique for sensitive interferometric detection of displacements," Phys. Lett. A 148, 8-16 (1990); M. W. Regehr, F. J. Raab, and S. E. Whitcomb, "Demonstration of power-recycled Michelson interferometer with Fabry-Perot arms by frontal modulation," Opt. Lett. 20, 1507-1509 (1995); R. Flaminio and H. Heitmann, "Longitudinal control of an interferometer for the detection of gravitational waves," Phys. Lett. A 214, 112-122 (1996).

20. R. W. P. Drever, J. L. Hall, F. V. Kowalski, J. Hough, G. M.
Ford, A. J. Munley, and H. Ward, "Laser phase and frequency stabilization using an optical resonator," Appl. Phys. B 31, 97-105 (1983).

21. B. Bhawal, in "Minutes of the control workshop," at Rome, Italy, B. Caron, ed., VIRGO Rep. VIR-LIS-LAP-1320-058 (Laboratoire d'Annecy de Physique des Particules, Annecy, France, 1996).

22. B. Bhawal, "Evolution of intracavity fields at a nonsteady state in a dual-recycled interferometer," Appl. Opt. 35, 1041-1045 (1996).

23. B. J. Meers, "Recycling in laser-interferometric gravitational-wave detectors," Phys. Rev. D 38, 2317-2326 (1988).

24. K.-X. Sun, M. M. Fejer, E. Gustafson, and R. L. Byer, "Sagnac interferometer for gravitational-wave detection," Phys. Rev. Lett. 76, 3053-3056 (1996). 